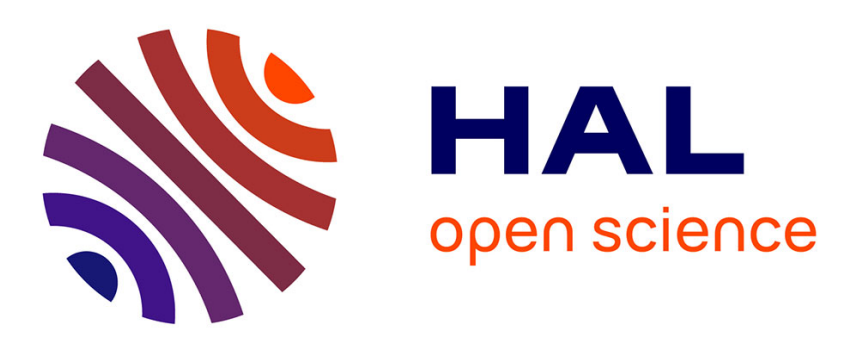

\title{
Simulation of Io's Auroral Emission: Constraints on the Atmosphere in Eclipse
}

Lorenz Roth, Joachim Saur, Kurt D. Retherford, Darrell F. Strobel, John R. Spencer

\section{- To cite this version:}

Lorenz Roth, Joachim Saur, Kurt D. Retherford, Darrell F. Strobel, John R. Spencer. Simulation of Io's Auroral Emission: Constraints on the Atmosphere in Eclipse. Icarus, 2011, 214 (2), pp.495. 10.1016/j.icarus.2011.05.014 . hal-00786874

\section{HAL Id: hal-00786874 https://hal.science/hal-00786874}

Submitted on 11 Feb 2013

HAL is a multi-disciplinary open access archive for the deposit and dissemination of scientific research documents, whether they are published or not. The documents may come from teaching and research institutions in France or abroad, or from public or private research centers.
L'archive ouverte pluridisciplinaire HAL, est destinée au dépôt et à la diffusion de documents scientifiques de niveau recherche, publiés ou non, émanant des établissements d'enseignement et de recherche français ou étrangers, des laboratoires publics ou privés. 


\section{Accepted Manuscript}

Simulation of Io's Auroral Emission: Constraints on the Atmosphere in Eclipse

Lorenz Roth, Joachim Saur, Kurt D. Retherford, Darrell F. Strobel, John R.

Spencer

PII:

S0019-1035(11)00179-5

DOI:

10.1016/j.icarus.2011.05.014

Reference:

YICAR 9821

To appear in:

Icarus

Received Date: $\quad 18$ January 2011

Revised Date: $\quad 10$ May 2011

Accepted Date: $\quad 12$ May 2011

Please cite this article as: Roth, L., Saur, J., Retherford, K.D., Strobel, D.F., Spencer, J.R., Simulation of Io's Auroral Emission: Constraints on the Atmosphere in Eclipse, Icarus (2011), doi: 10.1016/j.icarus.2011.05.014

This is a PDF file of an unedited manuscript that has been accepted for publication. As a service to our customers we are providing this early version of the manuscript. The manuscript will undergo copyediting, typesetting, and review of the resulting proof before it is published in its final form. Please note that during the production process errors may be discovered which could affect the content, and all legal disclaimers that apply to the journal pertain. 


\title{
Simulation of Io's Auroral Emission: Constraints on the Atmosphere in Eclipse
}

\author{
Lorenz Roth*,a, Joachim Saur ${ }^{\mathrm{a}}$, Kurt D. Retherford ${ }^{\mathrm{b}}$, Darrell F. Strobel ${ }^{\mathrm{c}}$, \\ John R. Spencer ${ }^{\mathrm{d}}$, \\ ${ }^{a}$ Institute of Geophysics and Meteorology, University of Cologne, Germany. \\ ${ }^{b}$ Southwest Research Institute, San Antonio, Texas, USA. \\ ${ }^{c}$ Department of Earth and Planetary Science, Johns Hopkins University, Baltimore, \\ Maryland, USA. \\ ${ }^{d}$ Southwest Research Institute, Boulder, Colorado, USA.
}

\section{Abstract}

We study the morphology of Io's aurora by comparing simulation results of a three-dimensional (3D) two-fluid plasma model to observations by the highresolution Long-Range Reconnaissance Imager (LORRI) on-board the New Horizons spacecraft and by the Hubble Space Telescope Advanced Camera for Surveys (HST/ACS). In 2007, Io's auroral emission in eclipse has been observed simultaneously by LORRI and ACS and the observations revealed detailed features of the aurora, such as a huge glowing plume at the Tvashtar paterae close to the North pole. The auroral radiation is generated in Io's atmosphere by collisions between impinging magnetospheric electrons and various neutral gas components. We calculate the interaction of the magnetospheric plasma with Io's atmosphere-ionosphere and simulate the auroral emission. Our aurora model takes into account not only the direct influ-

\footnotetext{
${ }^{*}$ Corresponding author

Email address: roth@geo.uni-koeln.de (Lorenz Roth)
} 
ence of the atmospheric distribution on the morphology and intensity of the emission, but also the indirect influence of the atmosphere on the plasma environment and thus on the exciting electrons. We find that the observed morphology in eclipse can be explained by a smooth (non-patchy) equatorial atmosphere with a vertical column density that corresponds to $\sim 10 \%$ of the column density of the sunlit atmosphere. The atmosphere is asymmetric with two times higher density and extension on the downstream hemisphere. The auroral emission from the Tvashtar volcano enables us to constrain the plume gas content for the first time. According to our model, the observed intensity of the Tvashtar plume implies a mean column density of $\sim 5 \times 10^{15} \mathrm{~cm}^{-2}$ for the plume region.

Key words:

Io, Jupiter, satellites, Atmospheres, structure

\section{Introduction}

2 Io probably possesses the densest and most species-rich atmosphere of the four Galilean satellites of Jupiter. Besides the main constituent sulfur ${ }_{4}$ dioxide various minor species, such as $\mathrm{S}, \mathrm{S}_{2}, \mathrm{O}, \mathrm{SO}, \mathrm{Na}, \mathrm{K}$ and $\mathrm{Cl}$, have 5 already been observed in the vicinity of Io. Two possible sources for the 6 atmosphere were discussed since its discovery. On the one hand volcanic venting as a direct source can create a neutral gas cloud around the satellite. On the other hand, sublimation of $\mathrm{SO}_{2}$ frost from the surface by sunlight 9 is considered a possible driver of the atmosphere (Lellouch et al., 2007). $10 \mathrm{SO}_{2}$ frost abundance is found to be correlated with active volcanic regions 11 (Douté et al., 2001). Thus, the surface distribution of the atmosphere is 
related to the distribution of the volcanic regions on global scales, no matter if sublimation or direct outgassing is the main source. Prior observations of $\mathrm{SO}_{2}$ suggested a volcanically driven atmosphere (e.g., Lellouch et al., 1992). Recent observations (Jessup et al., 2004; Moullet et al., 2010) and model results (Saur and Strobel, 2004) indicate that the sublimation driven part clearly dominates the direct outgassing.

The frequency of both the active volcanic regions and the paterae decreases with increasing latitude (Lopes-Gautier et al., 1999; Radebaugh et al., 2001) and observations of Lyman- $\alpha$ absorption in the atmosphere indicate that the $\mathrm{SO}_{2}$ gas is concentrated likewise at lower latitudes (Strobel and Wolven, 2001).Feaga et al. (2009) derived a map of $\mathrm{SO}_{2}$ column density, which shows a relatively sharp density decrease at approximately $30-45^{\circ}$ north and south and a maximum column density on the anti-jovian hemisphere, which was first noted by Jessup et al. (2004) and Spencer et al. (2005) and later confirmed by Moullet et al. (2010). Walker et al. (2010) investigated effects of plasma heating as well as surface frost, molecular residence time and surface temperature distribution in a sophisticated $\mathrm{SO}_{2}$ gas dynamics simulation, which includes sublimation and direct outgassing. The simulation results are compared to several observations with a backwards radiative transfer model in a companion paper (Gratiy et al., 2010) and basically confirm the previously derived atmospheric distributions assuming certain surface conditions. Considering results from modeling, millimeter, infrared and ultraviolet observations Lellouch et al. (2007) concluded that Io's atmosphere has a mean vertical column density of $\sim(1-5) \times 10^{16} \mathrm{~cm}^{-2}$, covering $50-70 \%$ of Io's day-side hemisphere. 
The evolution of the auroral emission, while Io passes through Jupiter's shadow, possibly provides very instructive information about the nature of the atmosphere. In eclipse the surface temperature drops and the sublimation of $\mathrm{SO}_{2}$ strongly decreases. Besides, no incident sunlight is reflected and thus solely the electron excited emission from the atmosphere is observable. Analyzing the aurora offers a possibility to determine the composition and distribution of Io's diverse atmospheric gas environment (Geissler et al., 2004).

Aurora (or airglow) is commonly defined as radiation caused by charged particles, which excite molecules or atoms in an atmosphere (Chamberlain and Hunten, 1987). In the case of Io, thermal electrons of the jovian magnetosphere rotate with Jupiter's magnetic field and thus constantly flow past the slowly orbiting satellite and excite the atmospheric gas. The emission covers a broad wavelength range including ultraviolet, visible and infrared wavelengths and can be attributed to the various abundant species in Io's atmosphere. Previous observations and analysis of Io's aurora offered insights into the satellite's atmosphere (e.g. Clarke et al., 1994; Roesler et al., 1999) .

Since the arrival of the Galileo probe at the jovian system, Io's auroral emission has been observed many times by ground-based and space telescopes (e.g., Roesler et al., 1999) as well as by on-board cameras of the Galileo (e.g., Geissler et al., 1999, 2001) and Cassini (e.g., Geissler et al., 2004) spacecrafts. Two of these observations are shown in Figure 1. Almost all observations are dominated by a key feature: bright spots close to the sub- and anti-jovian limb at low latitudes. These equatorial spots move up and down with the rocking background magnetic field of Jupiter (Retherford 
et al., 2000). Geissler et al. (2001) also observed enhanced visible aurora close to volcanic plumes. Numerical simulations of the aurora by Saur et al. (2000) revealed that due to a diverted plasma flow the energy of the electrons is preferentially deposited on the flanks of Io and thus more energy reaches dense atmospheric layers around the sub- and anti-jovian points on the surface (see also aurora features in Figure 2). Therefore the bright equatorial spots are generated there. A tilt of the magnetic field leads to a tilted distribution of the magnetospheric electrons and the equatorial spots are thus correlated with the background field. Futhermore, auroral emissions appear to be brighter on the hemisphere facing the plasma torus centrifugal equator than on the other hemisphere (Retherford et al., 2003). Moore et al. (2010) found the same north/south dependency for a wake emission features simulating OI $630.0 \mathrm{~nm}$ emission observed by the HST Wide Field and Planetary Camera 2.

When Io enters Jupiter's shadow, two opposing effects control the evolution of this auroral radiation (Saur and Strobel, 2004). The atmospheric gas partly freezes out and thus less neutral gas can be excited by the ambient plasma. On the other hand, a decrease of atmospheric density after eclipse ingress leads to a decreasing interaction strength. The deflection and cooling of the plasma flow is lower and the streamlines of the electrons are less divergent. Saur and Strobel (2004) calculated the response of Io's electrodynamic interaction and radiation to a temporal change in the atmosphere. Depending on the total atmospheric decay, they find three qualitatively different scenarios with two of them including a transient post-eclipse brightening. Generally, the total emission intensity in eclipse was found to be lower than 
out of eclipse . Retherford (2002) investigated the variation of key features of the eclipse aurora using HST Space Telescope Imaging Spectrograph (STIS) observations and inferred a reduction factor of $\sim 1.5$ to 2 for the emission close to Io during eclipse compared to the sunlit atmosphere. Geissler et al. (2004) in turn investigated the auroral emission during eclipse at different wavelengths with Cassini filter observations. Comparing the observed brightness of an equatorial spot with modeled intensities they derived mixing ratios for various gases, which contribute to the equatorial emission, such as $\mathrm{O}, \mathrm{S}, \mathrm{Na}$ and K. Limb glows, which are attributed to minor components, indicate that O, Na and K are abundant all over the surface (Retherford, 2002; Geissler et al., 2004). Based on these eclipse observations Geissler et al. (2004) finally conclude that Io's atmosphere must be at least partly sustained directly by volcanism. Recent Monte Carlo simulations of the response to eclipse of the neutral gas by Moore et al. (2009) indicate a strong dependence on the abundance of non-condensable species during atmospheric collapse. In case of a mole-fraction of 0.35 of non-condesable gas a near-surface diffusion layer forms and the $\mathrm{SO}_{2}$ density decreases only slowly and does not drop below 0.18 of the initial column density during an eclipse event.

During the flyby of the New Horizons probe in 2007, the auroral emission was observed by the on-board camera LORRI (Spencer et al., 2007b) and by the Hubble Space Telescope Advanced Camera for Surveys (HST/ACS) (Retherford et al., 2007) (Figure 3a-c). The LORRI camera provides images with high resolution comparable to Galileo SSI Io eclipse observations and reveals new details, such as the glowing plume of the Tvashtar volcano close to the North pole. This allows a detailed investigation of the aurora 
morphology. With the simultaneous HST/ACS observation we are able to compare aurora morphologies from two different viewing geometries.

In our work we evaluate these observations regarding the morphology and the total intensity. Therefore we use a numerical model to calculate the interaction of the upstream plasma with Io's atmosphere-ionosphere and the auroral emission from the atmosphere. This method ensures that we take into account both effects, which influence the auroral response to an atmospheric collapse during an eclipse event, as described above in this section. Investigating various atmospheric distributions, we derive one atmospheric distribution in eclipse, which is able to explain the observed intensity and morphology of the two LORRI observations and the HST/ACS image at the same time. Furthermore we infer the gas content of the Tvashtar plume. The constraints on the equatorial atmosphere and on the plume provide an estimation of the atmospheric collapse and a contribution of volcanic outgassing to the atmosphere.

\section{Observations}

In February and March 2007 the New Horizons probe passed the jovian System on its way to Pluto. The closest approach to Io occurred on 28 February at a distance of $2.24 \times 10^{6} \mathrm{~km}$. Io was observed several times while passing through Jupiter's shadow (Retherford et al., 2007). During two of the eclipse passages LORRI, a narrow-angle high resolution camera on-board the spacecraft, took spectacular images of the auroral emission at visible and infrared wavelengths (Spencer et al., 2007a,b). According to the notation of Retherford et al. (2007) these two eclipse occasions will be denoted Ieclipse03 
and Ieclipse04. During the Ieclipse03 event simultaneous spatially resolved observations of Io's far-ultraviolet aurora have been achieved by the Advanced Camera for Surveys Solar Blind Channel (ACS/SBC) on the Hubble Space Telescope. Characteristics of the eclipse occasions and the LORRI and HST/ACS observations are given in Tables 1 and 2. The geometry of the observations is depicted in Figure 2.

The NH/LORRI observations during eclipses Ieclipse04 and Ieclipse03 are shown in Figures $3 \mathrm{a}$ and $3 \mathrm{~b}$ (in the order that they are discussed in the paper). The spatial resolution of LORRI is $4.96 \mu \mathrm{rad}$ per pixel (Cheng et al., 2008). For Ieclipse03 this corresponds to a pixel size of $\sim 15 \mathrm{~km} \times 15 \mathrm{~km}$ ( $\sim 200$ pixels per Io diameter). During Ieclipse04 LORRI was used in the $4 \times 4$ binning mode, which reduces the spatial resolution in both directions by 4 . The pixel size in that case is $\sim 56 \mathrm{~km} \times 56 \mathrm{~km}$, which is equivalent to $\sim 50$ pixels per Io diameter. The pass-band ranges from about $350 \mathrm{~nm}$ to $850 \mathrm{~nm}$. The measured radiation originates from various emitters. In the blue range $\mathrm{SO}_{2}$ and $\mathrm{S}_{2}$ emission bands are the main contributors. Excited atomic oxygen, atomic sodium and potassium are the major emitters in the green, red and infra-red range (Geissler et al., 2004). Atomic sulfur contributes only a minor fraction.

The conversion from detector counts to emission brightness depends on the wavelength dependent sensitivity of the respective instrument. Since the contributing emissions in the wavelength ranges of LORRI and ACS are not clearly defined, we used Pivot wavelengths of $607.6 \mathrm{~nm}$ (LORRI) and 143.7 nm (ACS) to convert to Rayleighs (brightness). To validate this method we used exemplary modeled emission spectra including various emitters $\left(\mathrm{SO}_{2}\right.$, 
$\mathrm{O}, \mathrm{S}, \mathrm{Na}$ and $\mathrm{K}$ ) and calculated the brightness of the LORRI images using the sensitivity curve of Cheng et al. (2008). With this method the intensity differs less than $20 \%$ from the intensity derived with the Pivot wavelength.

We now describe and assign the essential features in the observation images. During the Ieclipse03 observation (Figure 3b) the sub-spacecraft coordinates are $\sim 310^{\circ} \mathrm{W}$ (west longitude, $0^{\circ}$ towards Jupiter) and $\sim 7^{\circ} \mathrm{S}$ (Southern latitude, $\vartheta=0^{\circ}$ in the orbital plane) in the Io-centric coordinate system. Hence, the sub-jovian upstream quadrant is visible during the observation. The brightest small spots on the disk appear to be thermal emission. The measured intensity corresponds to a black body temperature of approximately 1200 K (Spencer et al., 2007b). Fainter small spots are low-altitude gas emission. On the upstream side (right-hand limb) enhanced radiation is visible only within a relatively small region, which has been attributed to a volcanic hot spot located east of the Girru paterae and therefore named "East Girru" (Spencer et al., 2007b). Considering the geometry of the background magnetic field during Ieclipse03, which we discuss in detail below, the observed emission maximum around East Girru could also result from the magnetic field tilt and represent a shifted anti-jovian equatorial spot. The main part of anti-jovian spot probably is hidden behind Io. However, the East Girru emission appears to consist of one low and one high altitude emission part. Assuming that the plasma parameters do not vary discontinuously, the puzzling two part emission could originate from two different atmospheric species, as for example emission from $\mathrm{SO}_{2}$ close to the surface and from atomic oxygen after dissociation of $\mathrm{SO}_{2}$ at higher altitudes. Since a spectrally resolved observation is not available, this feature cannot be an- 
alyzed for different emission lines. Around the downstream (left-hand) disk edge there is a diffuse emission region of approximately $500 \times 1,000 \mathrm{~km}^{2}$. This region is the sub-jovian equatorial spot identified in previous observations (e.g., Roesler et al., 1999). Apart from the equatorial band there is small enhanced emission just above the limb close to the North pole. The bright area can undoubtedly be assigned to the huge Tvashtar plume, which was first seen by Cassini in December 2000 (Porco et al., 2003). Unlike most of Io's volcanoes, Tvashtar is located at a high latitude near Io's North pole. As the global atmosphere is relatively thin at higher latitudes, Tvashtar creates a locally enhanced neutral atmosphere, which can be investigated separately from the equatorial atmosphere.

In the Ieclipse04 image (Figure 3a) the Tvashtar plume is clearly evident above the limb. The bright area is similar to the plume size derived from sunlit observations with a height of about $350 \mathrm{~km}$ and full width of 1100 $\mathrm{km}$ (Spencer et al., 2007b). The geometry of this image (approx. 240 $\mathrm{W} 3^{\circ} \mathrm{S}$ ) allows observation of both equatorial spots. While the sub-jovian spot (left-hand) is restricted to a small area, the anti-jovian spot extends further on the disk. The extent to which a few dozen lower intensity features located at known volcanic vent locations may include atmospheric emissions combined with the thermal emissions is yet to be determined. For Ieclipse04 the deviation from the upstream view at $270^{\circ} \mathrm{W}$, where both spots should be visible and similar in brightness, is $\sim 30^{\circ}$. Note that in the case of Ieclipse03 the deviation from the upstream view is only $\sim 10^{\circ}$ more, but there is no clear anti-jovian spot observable (see description above).

The average of four consecutive exposures of Io in eclipse by HST/ACS 
(Retherford et al., 2007) is displayed in Figure 3c. This image includes emission in the $125 \mathrm{~nm}$ to $190 \mathrm{~nm}$ band-pass, which originates mostly from excited atomic sulfur and oxygen. $\mathrm{O}$ and $\mathrm{S}$ are expected to resemble $\mathrm{SO}_{2}$ in the region of interest for most of the analyzed emissions, although larger differences might occur at higher altitudes. The viewing angle from HST is $\sim 344^{\circ} \mathrm{W}$ and $\sim 0^{\circ} \mathrm{N} / \mathrm{S}$. Thus the complete sub-jovian side is visible, the plasma flow is directed from right (upstream) to left (downstream). The morphology is dominated by three features. The brightest area on the disk corresponds to the sub-jovian equatorial spot. The next brightest emission to the left of Io can either be attributed to the far end of the equatorial spots or originate directly in the wake of Io. And third, similar to the LORRI observation, the emission on the upstream (right-hand) side is considerably enhanced only above the East Girru region. Again, this enhancement can not definitely be assigned to a volcanic region, but might also originate from a smooth, continuous atmosphere and an inhomogeneous electron environment. As already mentioned, the $\mathrm{NH}$ Alice spectrograph additionally measured the total intensity of the two prominent oxygen multiplets at $130.4 \mathrm{~nm}$ and $135.6 \mathrm{~nm}$ and the sulfur multiplet at $147.9 \mathrm{~nm}$ during four eclipse events between 25 February and 3 March 2007 (see Table 3 and Retherford et al. (2007)).

Due to the tilt between Jupiter's dipole moment and Jupiter's rotation axis, the background field and the surrounding plasma density change while Io is moving up and down in the plasma torus during a synodic rotation period of Jupiter $\left(\mathrm{T}_{\text {syn }} \approx 12.95 \mathrm{~h}\right.$ ) with respect to Io. It takes about two hours for Io to pass through Jupiter's shadow, which is approximately one sixth of the 
synodic period of the varying background field. All displayed observation images (Figures 3a-c) are combinations of several coaligned exposures during one eclipse event (averaging regions not contaminated with instrument scattered light), and thus include changes in the plasma environment between the exposures. Although we can not identify one exact observation geometry, we now roughly describe the plasma conditions during the LORRI and HST observations. We use an Io-centered coordinate system, where $z$ is Jupiter (Io) North, and $x$ is along the orbital direction of Io, i.e. approximately along the plasma flow (but rocks due to the tilt of the plasma torus). $y$ completes the system pointing roughly (Io's orbit is slightly eccentric) towards Jupiter (Figure 2). The three components of $\vec{B}$ calculated with the model of Connerney et al. (1998) for Jupiter's internal field are listed in Table 1.

During Ieclipse03 Io is above the plasma torus and reaches the maximum distance to the torus center shortly before egress. The $y$ component of the magnetic field vector is relatively large. The component in the direction of the orbital movement $\left(B_{x}\right)$ in turn is low. The angle between the magnetic field and the polar axis $(z)$ varies between $\sim 16^{\circ}$ and $\sim 19^{\circ}$. During Ieclipse04 Io passes through the torus center from North to South. Accordingly, the $y$ component of $\vec{B}$ is lower and the $x$ component somewhat larger. The tilt of $\vec{B}$ to the polar axis is around $10^{\circ}$. Furthermore, the ambient plasma density presumably is higher during Ieclipse04 in the torus center than during Ieclipse03, when Io is far away from the center (Bagenal, 1994). The field vectors in the $x y$ plane during mid-eclipse are shown in Figure 2. The direction of the magnetic field for the observing geometry at mid-eclipse (Io in line with the center of the Sun and the center of Jupiter) is displayed in the 
lower right corner in Figures 3a-c.

In all observations five areas are highlighted with green frames, which we will separately analyze in this paper. First, two areas of $\sim 9.2 \times 10^{5} \mathrm{~km}^{2}$ around the equatorial spots, then $\mathrm{a} \sim 6.4 \times 10^{5} \mathrm{~km}^{2}$ sized region centered at the calculated position of the Tvashtar plume. The large boxes $\left(\sim 3.8 \times 10^{6}\right.$ $\mathrm{km}^{2}$ ) on the upper and lower edge of Io cover the polar areas, where the atmosphere is expected to be less dense and thus less emission is expected. We integrate the total emission within the boxes and normalize it to the covered area. This method allows us to investigate different features of the auroral morphology quantitatively.

For an appropriate theoretical description of the formation of Io's aurora, we first need to calculate the interaction of the plasma particles with Io's environment. As explained in section 1, the density and distribution of the atmospheric gas influence this interaction, i.e. the neutral gas controls the flow pattern and the temperature and density profiles of the electrons. Since the electrons generate the auroral emission, electron temperature and density are in addition to the atmospheric density the essential parameters for calculating the aurora. On the other hand, the distribution and local density of emitting gas particles is directly reflected by the intensity and morphology of the aurora. To calculate both effects self-consistently, we use a plasma model developed by Saur et al. (1999). 


\section{Model to interpret the observations}

\subsection{Plasma interaction model}

The model, which we use, was developed to simulate the plasma interaction of the satellites Europa (Saur et al., 1998) and Io (Saur et al., 1999). It has undergone several improvements subsequently. The simulation results provide explanations for several observed features of Io's plasma environment such as magnetic field signatures in the wake and the rotated Alfvén wing system. Furthermore, Saur et al. (2000) were able to explain the formation of the bright equatorial spots of Io's aurora. For the full set of equations and the numerical algorithms we refer the reader to Saur et al. (1999, 2002). In the following section we explain the basics of the model and the treatment of the two plasma parameters, which are essential for aurora simulation: the density and temperature of the electrons.

The simulation is developed in the $E, j$ approach of magnetohydrodynamics. The magnetic field is assumed to be a constant, homogeneous background field at all times. The electric conductivity parallel to the magnetic field is assumed to be infinite, so the parallel electric field vanishes. The validity of these assumptions are assessed in Neubauer (1998); Saur et al. (1999, 2002). With this model and its assumptions, Saur et al. (1999) were able to describe various aspects of the plasma interaction. In eclipse the local plasma interaction and thus the magnetic field perturbations are weaker than in the case of a sunlit atmosphere, i.e. the assumption of a homogeneous field is even more justified for our purposes. Galileo observations in sunlight revealed a magnetic field perturbation of more than $\frac{\Delta B}{B}=0.3$ (e.g. Kivelson et al., 
1996), which were reproduced by our model (Saur et al., 2002). For the interaction in eclipse the model results indicate that the perturbation of the magnetic field is lower by a factor of $2\left(\frac{\Delta B}{B} \approx 0.15\right.$ in eclipse). This justifies our assumption of a homogenous background magnetic field since the magnetic field environment is strongly dominated by the background field, while other plasma parameters such as velocity and electric field vary significantly due to the interaction with the atmosphere.

In the undisturbed upstream plasma the homogeneous electric field is given simply by $\vec{E}_{0}=-\vec{v}_{0} \times \vec{B}_{0}$. Inside Io's ionosphere a current system arises from the collisions between the plasma and neutral gas particles, which modify the electric field. The modified $\vec{E}$ field is calculated by a differential equation for the $2 \mathrm{D}$ electric potential in the plane perpendicular to $\vec{B}_{0}$ first derived by Wolf-Gladrow et al. (1987). The electric potential and thus the electric field around Io are calculated from the ionospheric Hall and Pedersen conductances as well as the Alfvén conductance. The undisturbed plasma velocity $\vec{v}_{0}$ is assumed to be perpendicular to $\vec{B}_{0}$. Neglecting inertia and pressure, the electron velocity $\vec{v}_{e}(x, y)$ in the plane perpendicular to $\vec{B}_{0}$ can be derived directly from the electric field. To simulate the auroral emission we need to calculate the properties of the thermal electron population in the vicinity of Io, which excite the aurora. Moving with the electron flow, the evolution of the density as well as the temperature of the electrons can be calculated as described below.

The coordinate system of the simulation corresponds to the system that we defined in section 2. The magnetic field however is constant and always anti-parallel to the $z$ axis and the plasma flow $v_{0}$ is parallel to $x$. Thus, we 
take into account neither changes of the inflow direction nor the rocking of the magnetic field due to the tilt of $9.6^{\circ}$ of the jovian dipole field axis with respect to the jovian rotation axis. The geometry is discussed in detail in section 3.4.

The evolution of the electron density $n_{e}$ is described by the following continuity equation:

$$
\frac{d}{d t} n_{e}=f_{i o n} n_{e} n_{S O_{2}}+k_{h e e} n_{S O_{2}}-\alpha n_{e}^{2}
$$

The first term on the right hand side describes (single) ionization by electron impact on neutral gas $n_{\mathrm{SO}_{2}}$ due to thermal electrons (Sittler and Strobel, 1987) with the temperature dependent collisional ionization rate $f_{\text {ion }}$.

With the second term we account for ionization by kappa-distributed energetic bidirectional electrons $\left(k_{h e e}\right)$ observed in Io's vicinity (e.g., Williams et al., 1999). The electron energies in the beams span the range from keV to hundreds of keV (Williams et al., 1996; Frank and Paterson, 2002). According to the simulated electron beam morphology by Jacobsen et al. (2010) and the observations during various Galileo flybys (Williams and Thorne, 2003; Frank and Paterson, 2002) we assume a spatial distribution of the high energetic electrons given by

$$
f_{\text {hee }}=f_{\text {hee }, 0}[\tanh (3 x+1)+1] \exp \left(-10 y^{6}\right) \cdot c(x, y)
$$

with

$$
\begin{array}{ccc}
c=1 & \text { for } & x^{2}+y^{2}>1 \mathrm{R}_{\mathrm{Io}} \\
c=0.5 & \text { for } & x^{2}+y^{2} \leq 1 \mathrm{R}_{\mathrm{Io}} .
\end{array}
$$

330 331

Thus, the beams are assumed to be relatively narrow (width in $y$ direction: $\sim 1.4 \mathrm{R}_{I o}$ ) but to extend far into the wake. However, outside the atmosphere 
(e.g. in the far wake), where the neutral density vanishes, no ionization can take place anyway. Using the geometrical factor $c(x, y)$ we take into account that directly above (below) Io the electron beam from the South (North) is shielded by the satellite. For the energy flux we use the derived value by Saur et al. (2002). Due to the absence of observations and models of electron beams when Io is eclipse, we note that expression (2) is derived from observations and models when Io is in sun light.

The third term on the RHS of equation (1) describes the loss due to recombination with a rate $\alpha$. For the adopted parameter values we refer the reader to Saur et al. (1999, 2002).

As the electron velocity in the model is simply given by the $\vec{E} \times \vec{B}$-drift, there is no plasma movement along $\vec{B}$ included. But, in fact plasma transport along $\vec{B}$ does occur, particularly in the wake of Io. The only electron source in the wake is the newly ionized population through the electron beams. For a low density atmosphere as in eclipse the ionization by electron beams is mainly confined to the region close to the equator, where the atmosphere is densest. Altogether, the wake region will be rather void, if movement along $\vec{B}$ is neglected. However, parallel movement due to the pressure gradient in the wake of Io possibly fills the relatively void regions in the downstream area and might thus enhance the aurora in the downstream region. To account for the parallel movement, we modified the model assuming that the plasma particles move along $\vec{B}$ with thermal velocity $v_{t h}$.

The total time derivative on the left hand side of (1) can be written as the partial time derivative and the convective term $\vec{v}_{e} \cdot \nabla n_{e}$. Separating the flow in the $x y$ plane from the movement along $z\left(\right.$ i.e. $\left.\vec{v}_{e} \cdot \nabla n_{e}=\vec{v}_{\perp} \cdot \nabla_{\perp} n_{e}+\vec{v}_{\|} \cdot \nabla_{\|} n_{e}\right)$ 
we rewrite the continuity equation as

$$
\vec{v}_{e}(x, y) \cdot \nabla_{\perp} n_{e}=f_{i o n} n_{e} n_{S O_{2}}+k_{h e e} n_{S O_{2}}-\alpha n_{e}^{2}-v_{t h} \nabla_{\|} n_{e}
$$

$\vec{v}_{\perp}$ is the electron flow $\vec{v}_{e}(x, y)$ and the parallel flow $\vec{v}_{\|}$along $z$ is approximated by the thermal velocity $v_{t h}$. Thus, following the $2 \mathrm{D}$ electron flow $\vec{v}_{e}(x, y)$ a parallel flow as loss or production term depending on the density gradient along $z$ is calculated. Numerically, we consider the net flow from or to both the grid cell above and below the current position. The flow direction is determined by the sign of the respective electron density gradient between the cells. This description is somewhat similar to a diffusive process. The thermal parallel velocity $v_{t h}$ is assumed to be the ion sound speed, as the inertia of the ions mainly determines the movement of the plasma. The ion sound speed is approximately half of the undisturbed relative flow velocity of the plasma (Kivelson et al., 2004), so the flow fills the wake at an angle of approximately $\arcsin \left(\frac{v_{t h}}{v_{e}(x, y)}\right) \geq 30^{\circ}$. The propagation along $\vec{B}_{0}$ enables the plasma to fill the wake of Io, which is important to explain the observed auroral emission in the downstream region. Expanding into low density regions such as Io's wake the plasma can be accelerated to velocities higher than the thermal velocity (Samir et al., 1983). By the assumption of a parallel movement with $v_{t h}$, the propagation speed into the wake might thus be underestimated.

The second important plasma quantity for aurora simulation is the thermal energy or temperature of the electrons, $T_{e}$. The temperature evolution is given by

$$
\begin{array}{r}
\frac{3}{2} k_{B} n_{e} \frac{d T_{e}}{d t}=-\left(\epsilon_{\text {ion }} f_{\text {ion }}+\epsilon_{\text {dis }} f_{\text {dis }}+\epsilon_{\text {rot }} f_{\text {rot }}+\epsilon_{\text {vib }} f_{\text {vib }}\right) \cdot n_{e} n_{S_{2}} \\
-\frac{3}{2} k_{B} T_{e}\left(f_{\text {ion }} n_{e} n_{S O_{2}}+k_{\text {hee }} n_{S O_{2}}\right)-\nabla Q_{\text {flux }} .
\end{array}
$$




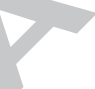

379

We account for cooling by inelastic collisions between the magnetospheric electrons and the atmosphere, including ionization, dissociation, and rotational and vibrational excitation of neutral $\mathrm{SO}_{2}$. Each process $\kappa$ is described by the rate $f_{\kappa}$ and the energy quantum $\epsilon_{\kappa}$. The newly added electrons resulting from the impact ionization processes are assumed to be cold. The adjustment to magnetospheric bulk temperature of these cold electrons leads to a decrease in temperature described by the next term on the right hand side. $k_{B}$ is the Boltzmann constant.

The heat flux $Q_{\text {flux }}$ is parametrized. We take advantage of the anisotropy of the thermal conductivity and assume no heat flow perpendicular to $\vec{B}$ due to the strong background magnetic field of $\sim 2000 \mathrm{nT}$ (Banks and Kockarts, 1973). Parallel to $\vec{B}$ the heat conduction is extremely high. We consider the electrons in a flux tube along $\vec{B}$ outside the atmosphere to adjust to one common temperature $T_{\text {out }}$ instantaneously. Deep inside the atmosphere the parallel thermal conductivity is lower, where the mixing ratio of neutral gas to plasma increases. When the flux tube passes through the atmosphere, we divide it in three parts, two outer parts $\left(T_{\text {out }}\right)$ and an inner part $\left(T_{\text {in }}\right)$. The inner part uniformly cools down due to the various collision processes described in equation 4 . The heat flow from the hotter outer part to the inner part $Q_{\text {flux }}$ is parametrized as sketched in Figure 4. Depending on the temperature dependent heat conductivity $\kappa\left(T_{i n}\right)$ and the temperature difference between the inner and outer parts a typical heat flow is calculated:

$$
<Q_{\text {flux }}>=<\kappa\left(T_{\text {in }}\right)>\frac{T_{\text {out }}-T_{\text {in }}}{R_{\text {typ }}} .
$$

The average heat transport is controlled by a typical distance $R_{t y p}$, which is set to the scale height of the atmosphere $(100 \mathrm{~km})$. The electron heat conduc- 
tivity $\kappa$ in a plasma with a fraction of neutral gas as function of the ambient temperature is given by equation (22.116) of Banks and Kockarts (1973) and depends on the momentum transfer cross section for elastic collisions of electrons with $\mathrm{SO}_{2}$ gas and the mixing ratio $n_{\mathrm{SO}_{2}} / n_{e}$. The temperature in the outer part $T_{\text {out }}$ decreases due to the heat flow to the cooler inner part, i.e. the outer parts serve as finite heat reservoirs and the energy of the entire flux tube is depleted. The energy capacity of these reservoirs corresponds to the electron content in the region along the magnetic field line above and below Io. For further details see Saur et al. (2002), Appendix A.

\subsection{Atmosphere model}

Based on several observations (see section 1) of Io's atmospheric distribution, we assume a dense atmospheric ring ranging from the equator to approximately $35^{\circ}$ North and South. At higher latitude a low density background $n_{b g}$ is assumed, with a ratio of $n_{b g} / n_{e q}=0.02$ for $\mathrm{SO}_{2}$. This distribution was calculated by Strobel and Wolven (2001) based on Lyman- $\alpha$ reflection observations. Here we investigate longitudinal asymmetries, such as differences between sub- and anti-jovian hemispheres reported by Spencer et al. (2005), Feaga et al. (2009) and Moullet et al. (2010) as well as a denser downstream atmosphere as inferred by Saur et al. (2002). The surface density $n_{s}$ is thus modeled by

$$
n_{s}(\vartheta, \varphi)=n_{b g}+\left(n_{e q}-n_{b g}\right)(1+\beta \cos (\varphi-\gamma)) \mathrm{e}^{-\left(\frac{\vartheta}{35^{\circ}}\right)^{6}}
$$

with the latitude $\vartheta$ and Jupiter oriented longitude $\varphi$ with $\varphi=0$ for direction to Jupiter and $\varphi=90^{\circ}$ in Io's orbital direction. $n_{e q}$ is the surface density at the equator, $\beta$ and $\gamma$ specify the strength and orientation of the longitudinal 
inhomogeneity and are fitted to match the observations. The number density declines exponentially with increasing altitude. The vertical structure is determined by the scale height $H_{s}$. We assume the scale height to vary with $\varphi$ according to the longitudinal variation of the surface density:

$$
H_{s}(\varphi)=H_{s, 0}(1+\beta \cos (\varphi-\gamma))
$$

The simultaneous variation of scale height and surface temperature allows an implementation of a larger difference in column density between two hemispheres with smaller local gradients than choosing a strongly varying surface density at a constant scale height. When the local density gradients are too large, the simulation becomes unstable.

For modeling the plasma interaction, we assume the atmosphere to consist solely of $\mathrm{SO}_{2}$, since it supposably is the by far main constituent (Lellouch et al., 2007). The surface scale height $H_{s, 0}$ in (7) for $\mathrm{SO}_{2}$ is set to $100 \mathrm{~km}$ (Saur et al., 1999). Near the surface the actual scale height is presumably lower in the range of tens of $\mathrm{km}$. But, for the low density eclipse atmosphere the plasma likely penetrates all atmospheric layers and the model accounts for integrated conductivities. At high altitutes a large scale height is expected. Thus, a scale height of $100 \mathrm{~km}$ represents an average scale height for Io's atmosphere and the interaction strength still constraints the global column density, since the crucial parameter for the interaction is the total atmospheric gas content.

The longitudinal distribution of the equatorial atmosphere in eclipse, which is modeled here, does not necessarily correspond to the longitudinal distribution at daytime seen around a full Io orbit. The fractional atmospheric collapse might vary with longitude due to different atmosphere 
and surface conditions or the position of the observer and the sun. For instance, the atmosphere may respond differently on the sub-Jupiter hemisphere, which is sunlit before eclipse ingress, than on the anti-jovian hemisphere, which is at night for several hours already before eclipse.

As the LORRI observations revealed a bright volcanic plume close to the North pole, we additionally implement a plume shaped density enhancement located at the Tvashtar paterae at $62^{\circ} \mathrm{N}$ and $122^{\circ} \mathrm{W}$ (Spencer et al., 2007b). The distribution within the plume is modeled by

$$
\begin{array}{r}
n_{V}(h, d)=n_{V, 0}\left(\exp \left[-\left(\left(\frac{h}{H_{V}}\right)^{2}+\left(\frac{d}{\sigma_{V}}\right)^{2}\right)^{3}\right]\right. \\
\left.-\exp \left[-\left(\left(\frac{h}{0.4 H_{V}}\right)^{2}+\left(\frac{d-d_{0}}{0.4 \sigma_{V}}\right)^{\frac{3}{2}}\right)^{3}\right]\right) \\
+30 n_{V, 0} \exp \left[-\left(\left(\frac{h}{0.1 H_{V}}\right)^{2}+\left(\frac{d}{0.05 \sigma_{V}}\right)^{2}\right)^{3}\right]
\end{array}
$$

where $n_{V, 0}$ is the density in the center of Tvashtar, $h$ the vertical distance from surface and $d$ the horizontal distance on the surface to the plume center. The height $H_{V}$ and width $\sigma_{V}$ are given by the observed plume extent in sunlight and are set to $H_{V}=360 \mathrm{~km}$ and $\sigma_{V}=550 \mathrm{~km}$. The subtracted exponential function (with height $0.4 H_{V}$ and width $0.4 \sigma_{V}$ ) in the second line roughly describes the expected low density region within the plume after Zhang et al. (2003). We also include a high density region above the vent by adding the third line in the equation. The plume model of Zhang et al. (2003) also indicates the formation of a canopy shock, which we do not account for in our simulation. $n_{V, 0}$ is the only free parameter of the plume. The three plume regions are marked in Figure 5, where a cross section through the volcano 
density and the column density above the plume are shown.

To model the radiated emission with the simulated electron densities and temperatures all the essential atmospheric constituents are considered individually. We will derive a mixing ratio $n_{\text {comp }} / n_{\text {total }}$ for the atmospheric components $\mathrm{O}, \mathrm{S}, \mathrm{Na}$ and K. For the simulation of the LORRI images the mixing ratio of the species is assumed to be identical in the equatorial atmosphere and in the plume. For atomic sulfur and oxygen the radial decrease is smaller than for $\mathrm{SO}_{2}$ (Wolven et al., 2001; Summers and Strobel, 1996). The Cassini observations of Geissler et al. (2004) revealed O assigned emission at higher surface distances than the emission assigned to $\mathrm{SO}_{2}$, see Figure 1a. This indicates a shallower decrease of $\mathrm{O}$ compared to $\mathrm{SO}_{2}$. For atomic sodium, HST observations of the NaI $589 \mathrm{~nm}$ line revealed a very shallow drop-off with increasing height (Retherford, 2002). Therefore, we assume a slightly larger scale height for these constituents of $H_{s, 0}(\mathrm{X})>H_{s, 0}\left(\mathrm{SO}_{2}\right)$ (see table 4). Moreover, we assume a higher $n_{b g} / n_{e q}$ ratio for atomic species, as the Cassini observations (Geissler et al., 2004) as well as HST observations (Retherford et al., 2000, 2003) show a clear limb glow all around Io for emission from atomic species.

\subsection{Emission simulation}

Assuming equilibrium, the local intensity of the stimulated emission of an atmospheric gas is calculated by

$$
i_{\lambda}(\vec{x})=f_{\lambda}\left(T_{e}(\vec{x})\right) n_{\text {gas }}(\vec{x}) n_{e}(\vec{x})
$$

where $f_{\lambda}$ is the gas specific rate, $n_{\text {gas }}$ the gas density and $n_{e}$ the density of the exciting electrons. Assuming spontaneous emission the emission rate cor- 
responds to the collisional excitation rate, which is given as an integral over the Maxwell-Boltzmann distribution, the electron velocity and the energydependent cross section for the collision of the exciting electrons with the neutral species. For optically thin emission lines a $2 \mathrm{D}$ emission pattern is given by the line-of-sight integral over the local intensities. Not all emissions can be considered optically thin. Therefore we estimate re-absorption for $\mathrm{Na}$ and K. All other emission lines are assumed to be optically thin in our analysis. Due to the long lifetime or small radiative decay rate of $k_{d}=0.00681$ $\mathrm{s}^{-1}$ of the $\mathrm{O}\left({ }^{1} D\right)$ state, we include collisional quenching at low altitudes for the OI 630.0/636.4 nm emission. The lowered intensity $I$ is calculated with the Stern-Vollmer relationship

$$
I=I_{0} \frac{k_{d}}{k_{d}+k_{q}}
$$

where $k_{q}$ is the quenching rate and $I_{0}$ the intensity without quenching. For $k_{q}$ we use the estimated value of $1 \times 10^{-10} \mathrm{~cm}^{3} \mathrm{~s}^{-1}$ from Geissler et al. (2004). Quenching and radiative decay are equally probable when the $\mathrm{SO}_{2}$ number density is $6.8 \times 10^{7} \mathrm{~cm}^{-3}$.

The NH/LORRI observations cover wavelengths from 350 to $850 \mathrm{~nm}$. Within this range emission from $\mathrm{SO}_{2}$ bands and $\mathrm{S}_{2}$ bands as well as from atomic oxygen $(557.7 \mathrm{~nm}, 630.0 \mathrm{~nm}, 636.4 \mathrm{~nm}, 777.4 \mathrm{~nm}$ and $844.6 \mathrm{~nm})$, atomic sulfur $(772.5 \mathrm{~nm})$, atomic sodium $(588.9 / 589.6 \mathrm{~nm})$ and potassium $(767.0 \mathrm{~nm})$ are expected. The OI $777.4 \mathrm{~nm}$ and OI $844.6 \mathrm{~nm}$ lines contribute less than $1 \%$ to the total emission and thus can be neglected (Geissler et al., 2004). The electron impact excitation cross sections that we use for atomic oxygen emission are based on the laboratory measurements of Doering and Gulcicek (1989a,b) and Doering (1992). The cross sections for the sodium 
and potassium D lines were adopted from theoretical calculations by Kim (2001), which the author compares to experimental data from Enemark and Gallagher (1972). For further details on the cross sections and the associated rates see Geissler et al. (2004) and references therein. Cross sections for electron excitation of $\mathrm{S}_{2}$ are not measured, so we can only estimate the contribution of $\mathrm{S}_{2}$ emission roughly.

A large number of emission lines can be found in the pass-band of the HST/ACS (125-190 nm) observation, such as emission from atomic oxygen and sulfur as well as from sulfur ions (Ballester et al., 1987; Roesler et al., 1999) and also chlorine emission (Feaga et al., 2004). Since cross sections are not available for all of the lines, we solely analyze the oxygen multiplet at $135.6 \mathrm{~nm}$ and the sulfur multiplet at $147.9 \mathrm{~nm}$, which presumably contribute most. Neglecting all the lower emissions in the pass-band, we can not compare the emission quantitatively with the ACS observation. A quantitative comparison also would necessitate an accurate analysis of optical depth of various lines. However, by simulating the OI $135.6 \mathrm{~nm}$ and SI 147.9 emission we are able to compare the model results to the ACS observation regarding the morphology and the relative intensities of the individually analyzed areas, since oxygen and sulfur generally are by far the main contributors in the observed wavelength range. The contribution of the OI $130.4 \mathrm{~nm}$ to the UV aurora is not clear yet and is therefore not considered here. For the sulfur emission at $147.9 \mathrm{~nm}$ we account for both the forbidden and allowed lines (Feaga et al., 2002). For both the SI147.9 nm and SI190.0 nm emission the adopted cross sections are based on the calculated collisions strengths from Zatsarinny and Tayal (2002). 


\subsection{Simulation setup}

A constant magnetic field and a 2D plasma flow perpendicular to $B_{0}$ are the basic assumptions of the theoretical approach. So it is not possible to fully consider the varying plasma conditions around Io in the simulation. In the model the constant background field is parallel to the $z$ axis and the upstream flow is in positive $x$ direction. Moreover, the model is symmetric with respect to the $x y$ plane given by $z=0$. For the implementation of the volcanic plume on the North pole, we run simulations with and without plume and combine the results. The influence of the plume on the aurora in the equator region appears to be negligible $(<1 \%)$. Note, the asymmetry of Io's atmosphere due to Tvashtar near Io's North pole generates also a small asymmetry in Io's plasma interaction. However, due to the small spatial extend of the plume compared to Io's diameter and the relative amount of the plume gas compared to the total gas of Io's atmosphere, the asymmetry in the plasma interaction is negligible in contrast to Enceladus' interaction, where the asymmetry plays an important role (Saur et al., 2007). We try to explain various observations with one atmospheric distribution, which is assumed to be symmetric around Io's equator due to the mentioned basic symmetry of the model around the equator. Hence, the orientation of the magnetic field and the upstream plasma flow are fixed in the model.

The actual plasma environment varies during a synodic rotation of Jupiter. The angle between the undisturbed plasma and the plane perpendicular to $\vec{B}_{0}$ varies only within $-3^{\circ}$ and $3^{\circ}$, which is negligible. The variation of the direction of the magnetic field with respect to the polar axis of Io is larger (up to $20^{\circ}$ ). As the simulation code is symmetric with respect to the equatorial 
plane, which is always assumed to be perpendicular to the background field, we are not able to investigate a tilt between the atmospheric ring and the orbital plane. Since the displayed observations are combinations of several exposures, the geometry is also varying for the single exposures. To minimize the effect of the simplified model geometry, we only analyze larger areas around the equatorial spots and Tvashtar quantitatively. Retherford et al. (2000) showed that the inclination of the spots is somewhat lower than the tilt of the magnetic field.

We account for the variation of the electron density in the upstream plasma. For Ieclipse03 simulation, where Io is far from the torus center we assume a lower density of $n_{e}=1900 \mathrm{~cm}^{-3}$, while during Ieclipse04 (Io crosses the torus center) the electron density is presumably higher and we use $n_{e}=3600 \mathrm{~cm}^{-3}$ (Gurnett et al., 2001b,a; Bagenal, 1994). The electron temperature of the upstream plasma is $T_{e, 0}=5 \mathrm{eV}$ (Bagenal, 1994). For the initial velocity we assume the relative azimuthal velocity of the rotating plasma $\left(\vec{v}_{0}=57 \mathrm{~km} \mathrm{~s}^{-1}\right)$, the background field is set to $B_{0}=2050 \mathrm{nT}$.

The viewing geometry of the displayed $2 \mathrm{D}$ emission patterns is given by longitude $\varphi$ and latitude $\vartheta$, where $\vartheta=0^{\circ}$, when the observer is in the orbital plane, and $\vartheta=90^{\circ}$, when viewed from above the North pole.

As discussed previously in section 2, we compare the simulated images with the observations using the green frame regions in Figure 3.

\section{Results}

First, we derive the total gas content and its distribution, which are determined mainly by $\mathrm{SO}_{2}$ abundance. Thereafter we briefly describe our 
derived mixing ratios and distributions for atomic oxygen and sulfur as well as the trace elements sodium and potassium. The emission from the atomic species also contributes to the intensity and morphology of the LORRI simulations, so the derivation of the abundances for $\mathrm{SO}_{2}$ and those of the minor components are correlated to each other. Therefore, we obtain the best-fit atmosphere results from an iterative variation of both the total gas content and the mixing ratios of the minor species. Finally, we constrain the gas content of the Tvashtar plume.

\subsection{Equatorial Atmosphere}

The main benchmark for constraining the density of the atmospheric ring was the morphology and location of the equatorial spots. We assume the same longitudinal variations for $\mathrm{SO}_{2}$ and the minor components.

First, we analyze the brightness of the equatorial spots within the green boxes of the LORRI observations. In the LORRI Ieclipse04 observation (Figure 3a) the ratio of the anti-jovian to the sub-jovian spot brightness is $I_{\text {anti }} / I_{\text {sub }} \approx 1.8$. For LORRI Ieclipse03 (Figure $3 \mathrm{~b}$ ) the anti-jovian spot can not be identified clearly. The spot might be displaced toward the East Girru feature. The ratio for the equator centered boxes in Figure 3b is $I_{\text {anti }} / I_{\text {sub }} \approx 0.2$. We get the best morphology agreement with both observations for a model atmosphere with an average equatorial column density of $N_{\text {eq,av }}=2.3 \times 10^{15} \mathrm{~cm}^{-2}$. We find a longitudinal asymmetry in favor of the downstream side with the asymmetry parameters $\beta=\frac{1}{3}$ and $\gamma=90^{\circ}$. The column density on the upstream side is thus $N_{e q, u p}=1.0 \times 10^{15} \mathrm{~cm}^{-2}$, the downstream column density is $N_{\text {eq, down }}=4.0 \times 10^{15} \mathrm{~cm}^{-2}$. The simulated aurora morphologies corresponding to the LORRI images are displayed in 
Figures $3 \mathrm{~d}$ and $3 \mathrm{e}$, where the brightness ratios are $I_{\text {anti }} / I_{\text {sub }} \approx 2.1$ (Ieclipse04) and $I_{\text {anti }} / I_{\text {sub }} \approx 0.4$ (Ieclipse03). The derived atmospheric distribution also yields a morphology that is in good agreement with the HST Ieclipse03 observation on the sub-jovian side. Furthermore, the key features of previous eclipse observations by Retherford (2002) are reproduced by the simulation considering the respective observation geometries (not shown here). The derived eclipse column density corresponds to $\sim 10 \%$ of the column density for a sunlit atmosphere summarized in Lellouch et al. (2007) $\left(N_{\text {sun }}=(1-\right.$ 5) $\times 10^{16} \mathrm{~cm}^{-2}$ ). This day-side column density also coincides with the column densities derived by Saur et al. (2000) and Saur et al. (2002), where the same simulation model for a sunlit atmosphere interaction is applied. The parameters of the best-fit atmosphere are listed in Table 4. The resulting absolute simulated and measured intensities in Rayleighs (R) of the marked areas are listed in Table 5 for comparison. The relative intensities refer to the absolute values divided by the total intensity. The total intensity $I_{t o t}$ in Rayleighs (R) corresponds to the total measured emission, but averaged to the area of Io's disk.

In order to illustrate the formation of the aurora morphology we show results of the plasma interaction simulation for Ieclipse03 in Figure 6: the atmospheric electron temperature in the flux tubes (Fig. 6a), the electron density in the equatorial plane (Fig. 6b) and the electric current in the Northern Alfvén wing (Fig. 6c). Due to the relatively low density in the equatorial atmosphere the energy stored in the flux tubes is sufficient to keep the electron temperature in most regions between 4 and $5 \mathrm{eV}$, and above $1 \mathrm{eV}$ everywhere in the interaction region. The lowest temperature 
is found on the flanks, where the electron density is highest. These high density regions in the anti- and sub-jovian equatorial region mainly control the position of the aurora equator spots. Depending on the overall density of the equatorial atmosphere the region of the maximum ionospheric density on the flanks and thus the aurora spots are shifted along the flow direction: for a denser atmosphere the maxima form further upstream, for a lower atmospheric density the spots move downstream. The low atmospheric density and the gas plume around Tvashtar also cause a complex current pattern in the Alfvén wing. In addition to the usual current system in the Northern wing, i.e. sub-jovian downward and anti-jovian upward currents, contrarily oriented currents evolve on both sides within the outer usual system. Because of the low neutral density around the poles and the overall low atmospheric density, only on the flanks a dense ionosphere forms. Consequently, the current system is inhomogeneously short-circuited in the atmosphere. Two separate current systems form on the anti- and sub-jovian hemisphere respectively. Furthermore, a small "winglet" is generated around the plume of the Tvashtar volcano, which we further discuss in Section 4.3. Simulation results for a similar model setup but a dense global atmosphere $\left(N=6 \times 10^{16} \mathrm{~cm}^{-3}\right)$ can be found in Saur et al. (1999).

In the case of a lower atmospheric surface density and thus column density $\left(N_{e q, a v}<1 \times 10^{15} \mathrm{~cm}^{-2}\right)$ the formation of high electron density regions (ionosphere) is weaker. The electrons also cool down less, but this plays a minor role at the given atmospheric thickness, since the cooling is already relatively low for the best-fit atmosphere. In the case of a lower density and a resulting lower ionosphere the diversion of the plasma flow decreases as well. 
Thus the equatorial spots move closer to the surface and further downstream. In this case the sub-jovian (left-hand side) spot in Figure 3d disappears behind the limb. Furthermore, the total intensity of the simulated emission averaged to the disk becomes far too low compared to the observations.

In the case of a higher equatorial column density of $N_{\text {eq,av }}>3 \times 10^{15} \mathrm{~cm}^{-2}$ the electron density on the upstream hemisphere increases due to increasing impact ionization, the electron flow is increasingly diverted around the body, and the cooling of the electrons is stronger and less energy reaches the downstream hemisphere. The equatorial spots are centered at larger distance from Io and the emission on the upstream side gets stronger compared to downstream side, as the plasma in the wake is completely cooled down. Thus, for such a high column density the emission morphology is not in agreement with the observation.

Assuming a longitudinal asymmetry with a higher and denser atmosphere on the anti-jovian hemisphere, as inferred for the day-side atmosphere (Jessup et al., 2004; Spencer et al., 2005; Feaga et al., 2009), yields a much stronger anti-jovian spot at larger radial distance. This would imply clear visibility of the spot during Ieclipse03 in Figure 3b, where no emission on the antijovian limb is detected except for the East Girru region. Furthermore, during Ieclipse04 the anti-jovian spot would be brighter and at a considerably higher altitude above the surface than the sub-jovian in the LORRI observation (Figure3a). As the anti-jovian emission maximum is only slightly higher and very close to the limb, there is no indication for an atmospheric anti-subjovian asymmetry in eclipse. The non-appearance of an atmospheric bulge on the anti-Jupiter side could be explained by the fact that it is nighttime 
on the anti-jovian hemisphere before eclipse ingress. The response of the atmosphere to a 21-hour night compared to a 2-hour eclipse event is likely to be different and the anti-jovian atmospheric bulge might be reduced due to that. Numerical calculations by Wong and Smyth (2000) indicate that non-condensible species might even dominate the night-side atmosphere.

Instead, the simulation results imply a longitudinal asymmetry of the atmosphere with increasing scale height and surface density from upstream to downstream. Compared to a longitudinally symmetric distribution, the emission is shifted downstream, which leads to a lower intensity on the upstream hemisphere and increasing intensity in the wake. The simulation still predicts more radiation to be emitted on the upstream than on the downstream side, although the downstream emission is more located and thus peaks higher. The observing geometry of the LORRI observations does not allow a separation of emission from the two hemispheres, but the emission appears to be brighter in the downstream region. However, larger datasets containing a range of exposures observing both upstream and downstream generally confirm a higher emission on the upstream hemisphere (Oliversen et al., 2001; Retherford, 2002) in agreement with our simulation results.

In the HST/ACS eclipse observation (Figure 3c) on the upstream hemisphere (right limb) aurora is observable only around East Girru, the corresponding simulation (Figure 3f) yields clear emission on the limb of the upstream equatorial side. In this region the fluid approach and the assumption of a constant background field might not describe the behavior of the electrons with the required accuracy. A highly distorted and piled up magnetic field in this region may hinder the electrons to move along $\vec{B}$ into the 
atmosphere near the equator. The electrons with pitch angles close to $90^{\circ}$ are reflected when the $\vec{B}$ increases and they do not reach thicker atmosphere layers. Moore et al. (2010) obtained this effect in their particle simulation. However, flux tubes with large $\vec{B}$ due to the upstream pile up have a smaller cross section close to Io. As these flux tubes are connected to a larger cross section further away from Io, a smaller area is linked to a larger energy reservoir. The combination of the both effects require further investigation with kinetic models. In the downstream region, the large cavity behind Io may lead to a faster filling of the wake than we assumed. This would cause higher emission in the wake.

Nonetheless, the position and size of the sub-jovian spot and the wake feature are in good agreement for the simulation and the HST/ACS observation (Figures 3c and 3f). The so-called wake feature is composed of two components. First, emission that is stimulated by electrons, which emerge from ionization by highly energetic beams directly 'behind' Io. Additionally, the tail of the spots can contribute to what is observed as wake emission, depending on the exact viewing geometry. In our simulation the contribution from the flanks is stronger. HST observations with viewing longitude between $\sim 60^{\circ}$ and $\sim 70^{\circ}$ (Figure 3.7 in Retherford, 2002) also revealed brighter flanks than radiation directly in the wake.

The longitudinal positions of the simulated equatorial spots range from $\sim 5^{\circ}$ to $\sim 40^{\circ}$ downstream from the zero meridian, which is roughly sketched in Figure 2. This is also in agreement with the derived position by Retherford et al. (2000) for HST observations of the day-side atmosphere $\left(10^{\circ}-30^{\circ}\right)$. The modeled brightness ratio of the anti-jovian to the sub-jovian spot as a 
function of the longitude of the observer is shown in Figure 7. Additionally, we plotted the brightness ratio of the OI $135.6 \mathrm{~nm}$ multiplet from observations by Retherford (2002), when Io was sunlit. These day-side observations and the eclipse simulation should not be compared directly here, but it can be pointed out that the model reproduces the basic longitudinal distribution of the aurora. The observed and simulated ratios are in agreement on the upstream hemisphere. For an observing longitude $\varphi$ of $90^{\circ}$ (into the wake) and $270^{\circ}$ (upstream hemisphere) the ratio is $\sim 1$.

On the upstream side the ratio varies roughly linearly with $\varphi$. In a low density atmosphere, e.g. during an eclipse, we would expect the spots at smaller surface distances and thus the variation to be steeper than out of eclipse. The simulated slope might therefore be too gradual due to an overestimation of the scale height in the model, which is presumably lower than assumed in the model close to the surface (Strobel and Wolven, 2001). For a smaller scale height the spots move closer to the surface and this would imply a steeper slope of the spot ratio as a function of the longitude.

On the downstream side the relation is inverse for $80^{\circ}<\varphi<100^{\circ}$, because the elongated spots are slightly tilted inwards. Therefore the line-ofsight integration for $\varphi \approx 85^{\circ}$ (resp. $\varphi \approx 95^{\circ}$ ) is approximately parallel to the anti-jovian (sub-jovian) spot and the spot appears brighter. The ratios of the wake observations by Retherford (2002) can not confirm this, but generally reveal a brighter sub-jovian spot for $\varphi<90^{\circ}$ in agreement with the model results.

An absolute value for the altitude of the equatorial spots midpoints is not readily determined for the observations. First, the spot emission is elongated 
over several degrees of longitude or several $100 \mathrm{~km}$ and thus it does not have one common altitude. Moreover, due to the two dimensional geometry and the viewing angle of the observations an altitude can not be derived without knowing the exact longitudinal extent or position. Choosing a hypothetical viewing geometry, where the observer is located right above the North pole, we can identify the height of the maximum emission on the flanks above the limb for our simulation results. Depending on the emitting species and the density of the upstream electrons, the local emission peaks at altitudes between $\sim 150 \mathrm{~km}$ and $\sim 300 \mathrm{~km}$ with our best-fit atmosphere. For a higher atmospheric density, the spots would appear at higher altitudes, as mentioned above, but again, the model probably overestimates the scale height and thus the spot altitude for that case.

The observed overall aurora brightness is similar between Ieclipse04 $\left(I_{t o t}=\right.$ $62.6 \mathrm{kR})$ and Ieclipse03 $\left(I_{\text {tot }}=40.9 k R\right)$. This agrees with the simulation, in which the total intensity varies with upstream electron density. In both cases the modeled intensity differs from the observed one by a factor of $\sim 2$. This discrepancy $\left(I_{\text {obs }} / I_{\text {sim }} \approx 2\right)$ can be traced back either to plasma conditions or atmospheric properties. The principal factor that controls the total intensity is the electron energy, which is deposited in the atmosphere. The influence of atmospheric density on the total intensity is less significant because of the opposed effects of an increase of neutral gas abundance: More neutral gas can be excited and radiate, but stronger deflection and divergence of the plasma flow (Section 1 and Saur and Strobel (2004)). Assuming the equatorial gas ring to extend to higher latitudes (up to $45^{\circ}$ instead of $35^{\circ}$, see equation 4.1) 
the total intensity would be increased by $~ 15 \%$ in our simulation. However, the electron energy deposited in the atmosphere is close to maximum for our derived atmosphere. So, the difference between observation and simulation is probably caused by differences in the plasma environment, of which no simultaneous measurements are available.

For instance, during the Ieclipse04 event Io passed through the torus center and thus possibly through very high plasma density regions. An even higher upstream plasma density than we assumed, would provide more energy and generate accordingly more radiation than calculated with our input parameters. Additionally, magnetic field aligned electron fluxes of higher energies $(\sim \mathrm{keV})$ can possibly excite auroral emission. Michael and Bhardwaj (2000) estimated a possible contribution to the aurora generation from energetic field-aligned electrons observed by the Galileo plasma instrument (Frank and Paterson, 1999). They found that the modeled emission is on the same order of brightness as the HST observations by Roesler et al. (1999), but do not consider the limited spatial distribution of the observed field aligned beams. Oliversen et al. (2001) measured short term (§10 min) intensity variations in the OI $630.0 \mathrm{~nm}$ line and ascribed this to a time-variable energy flux of field-aligned non-thermal electrons. Another possible reason for the discrepancy of simulation and observation are additional emitters in the wavelength range of the LORRI pass-band, which we do not account for in the simulation. Emission from $\mathrm{S}_{2}$ is expected at near-UV and blue visible wavelengths (Geissler et al., 2004), but not simulated due to the lack of exact electron impact cross sections. Since the morphology and position of the spots are to major parts determined by the absolute column density and not 
by the composition of the atmosphere, the abundance of $\mathrm{S}_{2}$ can be neglected here. In the Ieclipse03 LORRI image thermal emission around volcanic hot spots clearly contributes to the observed radiation, but is not simulated. Besides from these physical reasons, systematic uncertainties in the LORRI images might be large due to difficulties when compiling several exposures. Systematic errors such as copious instrument scattered light possibly leads to an under- or overestimation of the brightness.

\subsection{Minor components}

Since the LORRI observations do not spectrally resolve the emission, we compare our simulation results with a range of spectral observations of various emission lines resulting from oxygen, sulfur, sodium and potassium to constrain the abundance of these species. We are aware of the limitations of using observations made by different telescopes or cameras at different times, as for example long term variations and differing signal-to-noise ratios. But still, the measured intensities of the various observations differ not more than by a factor of 5 and are thus still a good way to derive abundances and check the derived atmospheric distribution with a wider range of observations. Some emission lines were frequently observed in the past, such as the oxygen multiplets OI $135.6 \mathrm{~nm}$ and OI $630.0 \mathrm{~nm}$. In that case, we preferably used the more recent observations. For both lines, modeled intensity and morphology are in good agreement with the observations (Retherford et al., 2007; Retherford, 2002; Bouchez et al., 2000; Geissler et al., 1999; Moore et al., 2010) using a mixing ratio of $12 \%$ of atomic oxygen to sulfur dioxide at the surface and a surface scale height of $150 \mathrm{~km}$. A comparison of the intensities for the analyzed emissions lines can be found in Table 3. 
To derive a sulfur mixing ratio we used the most recent observation of the SI 147.9 multiplet taken by the NH Alice spectrograph in 2007 as benchmark. The measurements revealed a varying total intensity during three eclipse events of $I=0.4-1.2 \mathrm{kR}$. Assuming a sulfur mixing ratio of $1.5 \%$ at the surface the model calculates a total intensity $I=0.6-1.1 \mathrm{kR}$. The intensity range in the model results is due to varying upstream electron density and variation of the viewing longitude. Comparisons with previous observations (Wolven et al., 2001; Feaga et al., 2002; Ballester et al., 1987) also yield a good agreement with the derived mixing ratio. Wolven et al. (2001) determined radial profiles for the OI 135.6 and SI 147.9 emission along the spatial axis of the aperture for HST/STIS observations. For the brightness close to Io averaged over the aperture width they find values between $\sim 0.1$ and $\sim 0.8$ $\mathrm{kR}$, where the emission from sulfur is found to be slightly higher than from oxygen. Depending on the sub-observer longitude, we calculate brightnesses averaged tangential to limb of $0.1-0.5 \mathrm{kR}$ for OI 135.6 and $0.3-0.9 \mathrm{kR}$ for SI 147.9 close to Io assuming the derived mixing ratios of oxygen and sulfur. The values match the observed brightness range of Wolven et al. (2001).

In case of the sodium emission we use the observed intensities in eclipse by Retherford (2002) and Bouchez et al. (2000) as reference. Compared to optically thin intensities the abundance of both $\mathrm{Na}$ and $\mathrm{K}$ need to be corrected by a factor of 3.5 and 3, respectively (Geissler et al., 2004). To achieve emission comparable to the observed intensities in the simulated sodium aurora an abundance of sodium bearing species of $0.12 \%$ (at the surface) has to be implemented, assuming a surface scale height of $150 \mathrm{~km}$. For potassium there are no direct measurements of $\mathrm{K}$ assigned emission lines in the 
close vicinity $\left(d<1 R_{i o}\right)$ of Io. Using the $\mathrm{Na} / \mathrm{K}$ ratio of 3.3 derived from infrared filter observations at 670 - $850 \mathrm{~nm}$ by Geissler et al. (2004) (0.04\% K abundance) the total intensity of the simulated KI $767.0 \mathrm{~nm}$ emission line is $I=2.2-4.3 \mathrm{kR}$. This is clearly lower than the total intensity of the infrared filter observations (Geissler et al., 2004), which provide an upper limit of 10.7 $\mathrm{kR}$.

All derived atmosphere parameters and the resulting equatorial column densities are summarized in Table 4 . The higher scale height of atomic species is in agreement with atmospheric models (e.g. Summers and Strobel, 1996). The higher background (polar) abundance for $\mathrm{O}, \mathrm{S}, \mathrm{Na}$ and $\mathrm{K}\left(n_{b g} / n_{e q}\right)$ is derived from the observed limb glow and polar emission for the respective emitter. The compared total intensities for all four analyzed components are listed in Table 3.

\subsection{Tvashtar Plume}

To constrain the plume density we analyze both the morphology and the intensity of the radiation inside the framed Tvashtar regions in Figure 3. During Ieclipse04 the whole plume of Tvashtar was in the field of view of LORRI, see Figure 3a. The plume is radiating rather uniformly with a maximum intensity in the plume center $\sim 220 \mathrm{~km}$ above the limb. The simulated absolute flux inside the framed volcano region, $I_{\text {Tvashtar }}$, is half of the observed flux from this region. Since for Ieclipse04 the simulated total intensity $I_{\text {tot }}$ also differs approximately by factor 2 from the observation, we compared the ratio $I_{\text {Tvashtar }} / I_{\text {tot }}$ with our simulation results. For Ieclipse04 the averaged emission intensity inside the Tvashtar region is almost equal to the disk-averaged total intensity, i.e. the ratio is $I_{\text {Tvashtar }} / I_{\text {tot }}=1.0$. Assuming a gas density 
of $n_{V, 0}=1.7 \times 10^{8} \mathrm{~cm}^{-3}$ in the main plume or an average column density of $N_{V}=5 \times 10^{15} \mathrm{~cm}^{-2}$ over the plume region the modeled plume aurora (Figure $3 \mathrm{~d})$ is in good agreement with the measurements $\left(I_{\text {Tvashtar }} / I_{\text {tot }}=0.9\right)$, see also Table 2. A small Alfvén wing is generated above Tvashtar due to the increased neutral and thus plasma density in the region, see Figure 6c. Yet, with the derived plume density, the electrons are still able to excite emission all over the plume (Figure 3d), since the plasma flow is diverted only moderately. The intensity maximum is found to be in the radial center at a limb distance of $\sim 180 \mathrm{~km}$ and plume emission appears to be rather uniformly. In the LORRI Ieclipse03 observation the upper edge of the glowing plume is visible just above the limb. The emission in the observation and simulated aurora match very well regarding morphology and relative strength.

For a lower plume density of $N_{V}<3 \times 10^{15} \mathrm{~cm}^{-2}$ the total modeled emission is too weak. For both LORRI observations the intensity ratio of plume and disk-averaged emission would be lower than the observed ratio by a least a factor of 2 . In the case of a higher plume density, $N_{V}>7 \times 10^{15} \mathrm{~cm}^{-2}$ the current system in and above the plume (see Figure 6c) becomes stronger. This leads to a stronger diversion of the electron flow around the plume region. The electrons deposit energy mostly on the upstream side of such a dense plume and the simulated aurora appears non-uniformly with a clear maximum at the upstream edge. On the other hand, the plume averaged emission intensity hardly increases with increasing plume density. The energy deposited all over the plume is almost at maximum for our best-fit plume density $N_{V}=5 \times 10^{15} \mathrm{~cm}^{-2}$, so that a denser plume does not induce a brighter auroral emission. 
Although the viewing angle of the HST/ACS Ieclipse03 image (Figure 3c) allows the whole plume to be visible by HST, only a very weak UV emission enhancement of $I_{\text {tvashtar }} / I_{\text {poles }} \approx 1.2$ was observed in the expected region. With the derived column density of $N_{V}=5 \times 10^{15} \mathrm{~cm}^{-2}$, our simulation predicts a $\sim 3$ times higher emission inside the Tvashtar region compared to the polar area around it. The pass-band of the HST observation includes mostly oxygen and sulfur emission lines. The lack of an emission enhancement around Tvashtar indicate a low abundance of $\mathrm{O}$ and $\mathrm{S}$ in the plume. With 3 times lower abundances of atomic sulfur $(\sim 0.5 \%)$ and oxygen $(\sim 4 \%)$ within the plume the $I_{\text {tvashtar }} / I_{\text {poles }}$ ratio matches approximately the HST/ACS observation.

As Tvashtar is considered a Pele-type plume, a high $\mathrm{S}_{2}$ abundance is expected. Observations of the Tvashtar plume in scattered light and absorption by Jessup and Spencer (2008) revealed a spectral behavior that is consistent with previous observations of the Pele plume. For Pele various $\mathrm{S}_{2} / \mathrm{SO}_{2}$ ratios between $\sim 1 \%$ and $30 \%$ have been obtained by Jessup et al. (2007). If dissociation of $\mathrm{SO}_{2}$ and $\mathrm{S}_{2}$ happens on time scales much larger than the average time of flight for the plume gas, the abundance of atomic sulfur and atomic oxygen in the plume will be lower than in an equatorial atmosphere, which is not solely of volcanic origin. Moses et al. (2002) show that the lifetimes for photolysis of $\mathrm{SO}_{2}$ or $\mathrm{S}_{2}$ producing $\mathrm{S}$ and $\mathrm{O}$ is in the range of hours, while ballistic flight times are on the order of 10 minutes. Using a thermodynamic model Fegley and Zolotov (2000) infer mixing ratios of atomic sulfur and oxygen to $\mathrm{SO}_{2}$ and $\mathrm{S}_{2}$ of the order of $10^{-2}$ and below. This implies a low abundance of $\mathrm{S}$ and $\mathrm{O}$ and would thus explain the low intensity in the UV 
range. On the other hand, with a low abundance of $\mathrm{O}$, which is also an essential emitter at visible wavelengths, the simulated plume intensity for the LORRI images would be reduced. Thus, the abundance of other species emitting in the LORRI wavelength must be higher to sustain the plume radiation. Another explanation for the non-visibility of the Tvashtar plume is the low resolution of the HST image, which possibly masks the local volcanic emission enhancement. A high resolution image in this UV range would offer a possibility to determine the exact abundance of atomic oxygen and sulfur in the plume.

The local density for a cut through the best-fit plume for the LORRI observations and the vertical column density as a function of the distance to the plume center $(d$ in $(8))$ are shown in Figure 5. Applying the model of a volcanic system developed by Kieffer (1982) and later adapted by Strobel and Wolven (2001) to the derived average column density of $N_{V}=5 \times 10^{15} \mathrm{~cm}^{-2}$ and the implemented plume size, Tvashtar appears to be a high temperature, low pressure volcano. The large size of the plume indicates relatively high particles velocities and thus a high temperature at the volcanic crater, which results from a high temperature $\left(T_{0} \approx 800 \mathrm{~K}\right)$ reservoir in the model system of Kieffer (1982). Our derived average column density is low compared to common plume models (e.g. Zhang et al., 2003) and would imply a low mean plume pressure of 0.1 nbar and correspond to the low pressure case (small plume size) discussed in Strobel and Wolven (2001).

Measurements and model calculations (e.g. by McGrath et al., 2000; Zhang et al., 2003) generally yield comparatively denser plumes for volcanoes close to the equator. Thus, our derived plume density and pressure appears 
to be relatively low. However, our method is hardly able to determine the plume density close to the surface accurately as the observed and simulated radiation is mostly emitted at a distance $>50 \mathrm{~km}$ from the ground. A higher localized density at very low altitudes might also not influence the plasma interaction strongly. If the density below $50 \mathrm{~km}$ increased exponentially over the whole plume area with an atmospheric scale height on the order of tens of km (as inferred by Strobel et al. (1994) for the equatorial atmosphere near the surface), the plume column density would go up by at least a factor of 2. For instance, a very low sticking coefficient for molecules that contact the surface could lead to a density increase at low altitudes.

The total content of the equatorial atmosphere, which we derived in section 4.1, equals $\sim 5-10$ times the total content of a volcanic plume with the plume density derived for Tvashtar. In other words, if we ruled out sublimation as possible atmospheric source, about 5-10 active volcanoes of the size of Tvashtar would be necessary to sustain the eclipse atmosphere. Pele-type plumes, such as Tvashtar, are the largest observed plumes and not more than 16 plumes have been observed so far (Geissler and Goldstein, 2007). Therefore it is unlikely that an atmosphere of the derived column density can be sustained solely by direct volcanic outgassing in eclipse, but there has to be an essential amount of gas species that do not condense during eclipse. The atmospheric density in sunlight is approximately ten times higher (Lellouch et al., 2007) than our derived equatorial atmosphere, so there would have to be 50-100 Tvashtar-sized active volcanoes to create such a dense atmosphere without sublimation. Although already more than 150 active regions have been discovered, above most regions no plumes have been ob- 
served (Lopes et al., 2004). Hence, direct volcanic outgassing is possibly able to sustain at least parts of the eclipse atmosphere, but cannot be considered as an essential source for the sunlit atmosphere.

\section{Summary and conclusions}

We modeled the auroral emission from the atmosphere while Io was in eclipse of Jupiter and investigated the effect of various atmospheric distributions on the intensity and morphology of the aurora. Our model results imply an atmospheric column density of $N_{\text {eclipse }}=(1-4) \times 10^{15} \mathrm{~cm}^{-2}$, i.e. when Io moves into Jupiter's shadow, its atmospheric density decreases down to $\sim 10 \%$ of the sunlit atmosphere assuming $N_{\text {sun }}=(1-5) \times 10^{16} \mathrm{~cm}^{-2}$ (Lellouch et al., 2007; Saur et al., 2000; Saur et al., 2002). Despite the density decrease, the atmosphere probably still covers most of the surface around the equator up to $\sim 35^{\circ}$ of latitude at these densities. With a smooth atmospheric ring and a low density ( $2 \%$ of equatorial density) at high latitudes we were able to reproduce the main features of the auroral emission, observed in various HST and spacecraft observations. Independently from the exact location of active volcanoes, sub-jovian and anti-jovian bright spots arise due to the diverted plasma flow. The various observed spot morphologies primarily result from the respective viewing geometry of the observer and the resulting visibility of the aurora features, such as the spots.

Compared to detailed atmospheric models (e.g. Walker et al., 2010), the inferred neutral distribution appears to be simplified. However, since the advantage of our model is the inclusion of the influence of a chosen atmospheric distribution on the plasma interaction and thus on the aurora generation, we 
did not consider the small scale variations of the equatorial atmosphere for numerical reasons but focused on the global structure.

Strong emission appearing in the downstream region of Io, which often is denoted as "wake emission", can be explained for major parts by flank emission extending far downstream. The derived variation of the brightness ratio between anti-jovian and sub-jovian spots in eclipse coincides with the observed variation in sunlight qualitatively. This implies that the aurora morphology is controlled by the plasma interaction also in eclipse and not only by the exact distribution of the atmospheric gas and/or the locations of volcanoes.

Analyzing various monochromatic observations we derived mixing ratios for minor components in the atmosphere: $12 \%$ atomic oxygen, $1.5 \%$ atomic sulfur and $0.12 \%$ sodium. The derived mixing ratios and the resulting column densities are in general agreement with previous observations and model results (Geissler et al., 2004; Wong and Smyth, 2000; Summers and Strobel, 1996), although our values are comparatively high.

For the Tvashtar plume we find a column density of $N_{V}=5 \times 10^{15} \mathrm{~cm}^{-2}$. This relatively low plume content supports the idea of an atmosphere that is sustained almost solely by sublimation. If a larger number of plumes of the Tvashtar size were active in the last decades, they likely would have been observed already. So far, 16 mostly smaller plumes have been observed (Geissler and Goldstein, 2007) and thus volcanic outgassing probably is not able to sustain a dense atmosphere as measured in sunlight.

Spectrally resolved observations with a resolution comparable to the LORRI images would provide a possibility to determine absolute values of 
the abundance of the various emitters. As the electron energy is distributed very inhomogeneously around Io, local inhomogeneities in the neutral gas abundance possibly influence the total intensity strongly. Spectral observations with a high spatial resolution like the LORRI images would allow a detailed analysis of various regions, taking into account the local electron parameters as well as the local neutral gas density and composition. For example, spectrally resolved observations of the Tvashtar plume emission could offer detailed information about the abundant species in this huge and outstanding plume.

\section{Acknowledgments}

1 L.R. and J.S. acknowledge support from BMWi and DLR, grant FKZ 50 OR 0805. K.D.R. appreciates support from NASA grant NNX09AU28G. D.F.S. acknowledges support from the New Horizons Mission through SWRI Contract No. 277043Q and NASA grant NNX10AB846. 


\section{References}

Bagenal, F., Jun. 1994. Empirical model of the Io plasma torus: Voyager measurements. J. Geophys. Res. 99 (A6), 11043-11062.

Ballester, G. E., Moos, H. W., Feldman, P. D., Strobel, D. F., Summers, M. E., Bertaux, J., Skinner, T. E., Festou, M. C., Lieske, J. H., Aug. 1987. Detection of neutral oxygen and sulfur emissions near Io using IUE. Astrophys. J. 319, L33-L38.

Banks, P. M., Kockarts, G., 1973. Aeronomy. Vol. A. Academic Press, San Diego, Calif.

Bouchez, A. H., Brown, M. E., Schneider, N. M., Nov. 2000. Eclipse Spectroscopy of Io's Atmosphere. Icarus 148, 316-319.

Chamberlain, J. W., Hunten, D. M., 1987. Theory of planetary atmospheres. An introduction to their physics and chemistry. Vol. 36 of International Geophysics Series. Academic Press Inc., Orlando, FL, USA.

Cheng, A. F., Weaver, H. A., Conard, S. J., Morgan, M. F., Barnouin-Jha, O., Boldt, J. D., Cooper, K. A., Darlington, E. H., Grey, M. P., Hayes, J. R., Kosakowski, K. E., Magee, T., Rossano, E., Sampath, D., Schlemm, C., Taylor, H. W., Oct. 2008. Long-Range Reconnaissance Imager on New Horizons. Space Science Reviews 140, 189-215.

Clarke, J. T., Ajello, J., Luhmann, J., Schneider, N., Kanik, I., Apr. 1994. Hubble Space Telescope UV spectral observations of Io passing into eclipse. J. Geophys. Res. 99, 8387-8402. 
Connerney, J. E. P., Acuña, M. H., Ness, N. F., Satoh, T., Jun. 1998. New models of Jupiter's magnetic field constrained by the Io flux tube footprint. J. Geophys. Res. 103, 11929-11940.

Doering, J. P., Dec. 1992. Absolute differential and integral electron excitation cross sections for atomic oxygen. IX - Improved cross section for the ${ }^{3}$ P_ ${ }^{1}$ D transition from 4.0 to 30 eV. J. Geophys. Res. 97, 19531-19534.

Doering, J. P., Gulcicek, E. E., Feb. 1989a. Absolute differential and integral electron excitation cross sections for atomic oxygen. VII - The ${ }^{3} \mathrm{P}-{ }^{1} \mathrm{D}$ and ${ }^{3} \mathrm{P}-{ }^{1} \mathrm{~S}$ transitions from 4.0 to $30 \mathrm{eV}$. J. Geophys. Res. 94, 1541-1546.

Doering, J. P., Gulcicek, E. E., Mar. 1989b. Absolute differential and integral electron excitation cross sections for atomic oxygen. VIII - The ${ }^{3} \mathrm{P}-{ }^{5} \mathrm{~S}^{0}$ transition (1356 A) from 13.9 to 30 eV. J. Geophys. Res. 94, 2733-2736.

Douté, S., Schmitt, B., Lopes-Gautier, R., Carlson, R., Soderblom, L., Shirley, J., Galileo NIMS Team, Jan. 2001. Mapping $\mathrm{SO}_{2}$ Frost on Io by the Modeling of NIMS Hyperspectral Images. Icarus 149, 107-132.

Enemark, E. A., Gallagher, A., Jul. 1972. Electron Excitation of the Sodium D Lines. Physical Review A 6, 192-205.

Feaga, L. M., McGrath, M., Feldman, P. D., Jun. 2009. Io's dayside $\mathrm{SO}_{2}$ atmosphere. Icarus 201, 570-584.

Feaga, L. M., McGrath, M. A., Feldman, P. D., May 2002. The Abundance of Atomic Sulfur in the Atmosphere of Io. Astrophys. J. 570, 439-446. 
Feaga, L. M., McGrath, M. A., Feldman, P. D., Strobel, D. F., Aug. 2004. Detection of Atomic Chlorine in Io's Atmosphere with the Hubble Space Telescope GHRS. Astrophys. J. 610, 1191-1198.

Fegley, B., Zolotov, M. Y., Nov. 2000. Chemistry of Sodium, Potassium, and Chlorine in Volcanic Gases on Io. Icarus 148, 193-210.

Frank, L. A., Paterson, W. R., 1999. Intense electron beams observed at Io with the Galileo spacecraft. J. Geophys. Res. 104, 28657-28670.

Frank, L. A., Paterson, W. R., Aug. 2002. Plasmas observed with the Galileo spacecraft during its flyby over Io's northern polar region. Journal of Geophysical Research (Space Physics) 107, 1220-1238.

Geissler, P., McEwen, A., Porco, C., Strobel, D., Saur, J., Ajello, J., West, R., Nov. 2004. Cassini observations of Io's visible aurorae. Icarus 172, $127-$ 140.

Geissler, P. E., Goldstein, D. B., 2007. Plumes and their deposits. Springer Praxis Books / Geophysical Sciences, pp. 163-192.

Geissler, P. E., McEwen, A. S., Ip, W., Belton, M. J. S., Johnson, T. V., Smyth, W. H., Ingersoll, A. P., Aug. 1999. Galileo Imaging of Atmospheric Emissions from Io. Science 285, 870-874.

Geissler, P. E., Smyth, W. H., McEwen, A. S., Ip, W., Belton, M. J. S., Johnson, T. V., Ingersoll, A. P., Rages, K., Hubbard, W., Dessler, A. J., Nov. 2001. Morphology and time variability of Io's visible aurora. J. Geophys. Res. 106, 26137-26146. 
Gratiy, S. L., Walker, A. C., Levin, D. A., Goldstein, D. B., Varghese, P. L., Trafton, L. M., Moore, C. H., May 2010. Multi-wavelength simulations of atmospheric radiation from Io with a 3-D spherical-shell backward Monte Carlo radiative transfer model. Icarus 207, 394-408.

Gurnett, D. A., Kurth, W. S., Persoon, A. M., Roux, A., Bolton, S. J., Dec. 2001a. An Overview of Galileo Plasma Wave Observations During the I31 and I32 Flybys of Io. AGU Fall Meeting Abstracts.

Gurnett, D. A., Persoon, A. M., Kurth, W. S., Roux, A., Bolton, S. J., Nov. 2001b. Electron densities near Io from Galileo plasma wave observations. J. Geophys. Res. 106, 26225-26232.

Jacobsen, S., Saur, J., Neubauer, F. M., Bonfond, B., Gérard, J., Grodent, D., Apr. 2010. Location and spatial shape of electron beams in Io's wake. Journal of Geophysical Research (Space Physics) 115 (A14), 4205-4214.

Jessup, K., Spencer, J. R., Mar. 2008. Detailed Analysis of the Tvashtar Plume Spectral Behavior. In: Lunar and Planetary Institute Science Conference Abstracts. Vol. 39 of Lunar and Planetary Inst. Technical Report.

Jessup, K. L., Spencer, J., Yelle, R., Dec. 2007. Sulfur volcanism on Io. Icarus $192,24-40$.

Jessup, K. L., Spencer, J. R., Ballester, G. E., Howell, R. R., Roesler, F., Vigel, M., Yelle, R., May 2004. The atmospheric signature of Io's Prometheus plume and anti-jovian hemisphere: evidence for a sublimation atmosphere. Icarus 169, 197-215. 
Kieffer, S. W., 1982. Dynamics and thermodynamics of volcanic eruptions - Implications for the plumes on Io. In: D. Morrison (Ed.), Satellites of Jupiter. pp. 647-723.

Kim, Y., Sep. 2001. Scaling of plane-wave Born cross sections for electronimpact excitation of neutral atoms. Physical Review A 64 (3).

Kivelson, M. G., Bagenal, F., Kurth, W. S., Neubauer, F. M., Paranicas, C., Saur, J., 2004. Magnetospheric interactions with satellites. pp. 513-536.

Kivelson, M. G., Khurana, K. K., Walker, R. J., Warnecke, J., Russell, C. T., Linker, J. A., Southwood, D. J., Polanskey, C., Oct. 1996. Lo's Interaction with the Plasma Torus: Galileo Magnetometer Report. Science 274, 396398.

Lellouch, E., Belton, M., de Pater, I., Paubert, G., Gulkis, S., Encrenaz, T., Aug. 1992. The structure, stability, and global distribution of Io's atmosphere. Icarus 98, 271-295.

Lellouch, E., McGrath, M. A., Jessup, K. L., 2007. Io's atmosphere. Springer Praxis Books / Geophysical Sciences, pp. 231-264.

Lopes, R. M. C., Kamp, L. W., Smythe, W. D., Mouginis-Mark, P., Kargel, J., Radebaugh, J., Turtle, E. P., Perry, J., Williams, D. A., Carlson, R. W., Douté, S., May 2004. Lava lakes on Io: observations of Io's volcanic activity from Galileo NIMS during the 2001 fly-bys. Icarus 169, 140-174.

Lopes-Gautier, R., McEwen, A. S., Smythe, W. B., Geissler, P. E., Kamp, L., Davies, A. G., Spencer, J. R., Keszthelyi, L., Carlson, R., Leader, 
F. E., Mehlman, R., Soderblom, L., The Galileo NIMS And SSI Teams, Aug. 1999. Active Volcanism on Io: Global Distribution and Variations in Activity. Icarus 140, 243-264.

McGrath, M. A., Belton, M. J. S., Spencer, J. R., Sartoretti, P., Aug. 2000. Spatially Resolved Spectroscopy of Io's Pele Plume and $\mathrm{SO}_{2}$ Atmosphere. Icarus 146, 476-493.

Michael, M., Bhardwaj, A., Oct. 2000. FUV emissions on Io: Role of Galileoobserved field-aligned energetic electrons. Geophys. Res. Lett. 27, 31373140.

Moore, C., Miki, K., Goldstein, D. B., Stapelfeldt, K., Varghese, P. L., Trafton, L. M., Evans, R. W., Jun. 2010. Monte Carlo modeling of Io's [OI] $6300 \AA$ and [SII] $6716 \AA$ auroral emission in eclipse. Icarus 207, 810833.

Moore, C. H., Goldstein, D. B., Varghese, P. L., Trafton, L. M., Stewart, B., Jun. 2009. 1-D DSMC simulation of Io's atmospheric collapse and reformation during and after eclipse. Icarus 201, 585-597.

Moses, J. I., Zolotov, M. Y., Fegley, B., Mar. 2002. Alkali and Chlorine Photochemistry in a Volcanically Driven Atmosphere on Io. Icarus 156, $107-135$.

Moullet, A., Gurwell, M. A., Lellouch, E., Moreno, R., 2010. Simultaneous mapping of $\mathrm{SO}_{2}, \mathrm{SO}, \mathrm{NaCl}$ in Io's atmosphere with the Submillimeter Array. Icarus 208 (1), 353-365. 
Neubauer, F. M., Sep. 1998. The sub-Alfvénic interaction of the Galilean satellites with the Jovian magnetosphere. J. Geophys. Res. 103 (E9), 19843-19866.

Oliversen, R. J., Scherb, F., Smyth, W. H., Freed, M. E., Woodward, R. C. J., Marconi, M. L., Retherford, K. D., Lupie, O. L., Morgenthaler, J. P., Nov. 2001. Sunlit Io atmospheric [OI] $6300 \AA$ emission and the plasma torus. J. Geophys. Res. 106, 26183-26194.

Porco, C. C., West, R. A., McEwen, A., Del Genio, A. D., Ingersoll, A. P., Thomas, P., Squyres, S., Dones, L., Murray, C. D., Johnson, T. V., Burns, J. A., Brahic, A., Neukum, G., Veverka, J., Barbara, J. M., Denk, T., Evans, M., Ferrier, J. J., Geissler, P., Helfenstein, P., Roatsch, T., Throop, H., Tiscareno, M., Vasavada, A. R., Mar. 2003. Cassini Imaging of Jupiter's Atmosphere, Satellites, and Rings. Science 299, 1541-1547.

Radebaugh, J., Keszthelyi, L. P., McEwen, A. S., Turtle, E. P., Jaeger, W., Milazzo, M., Dec. 2001. Paterae on Io: A new type of volcanic caldera? J. Geophys. Res. 106, 33005-33020.

Retherford, K. D., Dec. 2002. Io's aurora: HST/STIS observations. Ph.D. thesis, Johns Hopkins University, Baltimore, MD.

Retherford, K. D., Moos, H. W., Strobel, D. F., Aug. 2003. Io's auroral limb glow: Hubble Space Telescope FUV observations. Journal of Geophysical Research (Space Physics) 108, 1333-1341.

Retherford, K. D., Moos, H. W., Strobel, D. F., Wolven, B. C., Roesler, F. L., 
Dec. 2000. Io's equatorial spots: Morphology of neutral UV emissions. J. Geophys. Res. 105, 27157-27166.

Retherford, K. D., Spencer, J. R., Stern, S. A., Saur, J., Strobel, D. F., Steffl, A. J., Gladstone, G. R., Weaver, H. A., Cheng, A. F., Parker, J. W., Slater, D. C., Versteeg, M. H., Davis, M. W., Bagenal, F., Throop, H. B., Lopes, R. M. C., Reuter, D. C., Lunsford, A., Conard, S. J., Young, L. A., Moore, J. M., Oct. 2007. Io's Atmospheric Response to Eclipse: UV Aurorae Observations. Science 318, 237-240.

Roesler, F. L., Moos, H. W., Oliversen, R. J., Woodward, R. C., Retherford, K. D., Scherb, F., McGrath, M. A., Smyth, W. H., Feldman, P. D., Strobel, D. F., Jan. 1999. Far-ultraviolet imaging spectroscopy of Io's atmosphere with HST/STIS. Science 283 (5400), 353-357.

Samir, U., Wright, J. K. H., Stone, N. H., 1983. The expansion of a plasma into a vacuum: basic phenomena and processes and applications to space plasma physics. Reviews of Geophysics and Space Physics 21, 1631-1646.

Saur, J., Neubauer, F. M., Schilling, N., Nov. 2007. Hemisphere coupling in Enceladus' asymmetric plasma interaction. Journal of Geophysical Research (Space Physics) 112, A11209.

Saur, J., Neubauer, F. M., Strobel, D. F., Summers, M. E., Nov. 1999. Threedimensional plasma simulation of Io's interaction with the Io plasma torus: Asymmetric plasma flow. J. Geophys. Res. 104, 25105-25126.

Saur, J., Neubauer, F. M., Strobel, D. F., Summers, M. E., Sep. 2000. Io's 
ultraviolet aurora: Remote sensing of Io's interaction. Geophys. Res. Lett. 27 (18), 2893-2896.

Saur, J., Neubauer, F. M., Strobel, D. F., Summers, M. E., Dec. 2002. Interpretation of Galileo's Io plasma and field observations: I0, I24, and I27 flybys and close polar passes. Journal of Geophysical Research (Space Physics) 107, 1422-1439.

Saur, J., Strobel, D. F., Oct. 2004. Relative contributions of sublimation and volcanoes to Io's atmosphere inferred from its plasma interaction during solar eclipse. Icarus 171, 411-420.

Saur, J., Strobel, D. F., Neubauer, F. M., Aug. 1998. Interaction of the Jovian magnetosphere with Europa: Constraints on the neutral atmosphere. J. Geophys. Res. 103 (E9), 19947-19962.

Sittler, E. C., Strobel, D. F., Jun. 1987. Io plasma torus electrons - Voyager 1. J. Geophys. Res. 92, 5741-5762.

Spencer, J. R., Lellouch, E., Richter, M. J., López-Valverde, M. A., Jessup, K. L., Greathouse, T. K., Flaud, J., Aug. 2005. Mid-infrared detection of large longitudinal asymmetries in Io's $\mathrm{SO}_{2}$ atmosphere. Icarus 176, 283304.

Spencer, J. R., Stern, S., Retherford, K., Abramov, O., Reuter, D., Cheng, A., Weaver, H. A., Lunsford, A., Moore, J., Perry, J., Lopes, R. M., Kamp, L., New Horizons Science Team, Oct. 2007a. New Horizons Observes Io's Volcanic Activity. In: Bulletin of the American Astronomical Society. Vol. 38 of Bulletin of the American Astronomical Society. 
Spencer, J. R., Stern, S. A., Cheng, A. F., Weaver, H. A., Reuter, D. C., Retherford, K., Lunsford, A., Moore, J. M., Abramov, O., Lopes, R. M. C., Perry, J. E., Kamp, L., Showalter, M., Jessup, K. L., Marchis, F., Schenk, P. M., Dumas, C., Oct. 2007b. Io Volcanism Seen by New Horizons: A Major Eruption of the Tvashtar Volcano. Science 318, 240-243.

Strobel, D. F., Wolven, B. C., Jun. 2001. The Atmosphere of Io: Abundances and Sources of Sulfur Dioxide and Atomic Hydrogen. Astrophys. Space Sci. $277,271-287$.

Strobel, D. F., Zhu, X., Summers, M. F., Sep. 1994. On the vertical thermal structure of Io's atmosphere. Icarus 111, 18-30.

Summers, M. E., Strobel, D. F., Apr. 1996. Photochemistry and Vertical Transport in Io's Atmosphere and Ionosphere. Icarus 120, 290-316.

Walker, A. C., Gratiy, S. L., Goldstein, D. B., Moore, C. H., Varghese, P. L., Trafton, L. M., Levin, D. A., Stewart, B., May 2010. A comprehensive numerical simulation of Io's sublimation-driven atmosphere. Icarus 207, 409-432.

Williams, D. J., Mauk, B. H., McEntire, R. E., Roelof, E. C., Armstrong, T. P., Wilken, B., Roederer, J. G., Krimigis, S. M., Fritz, T. A., Lanzerotti, L. J., Oct. 1996. Electron Beams and lon Composition Measured at lo and in Its Torus. Science 274, 401-403.

Williams, D. J., Thorne, R. M., Nov. 2003. Energetic particles over Io's polar caps. Journal of Geophysical Research (Space Physics) 108, 1397-1403. 
1215

1216

Williams, D. J., Thorne, R. M., Mauk, B., Jul. 1999. Energetic electron beams and trapped electrons at Io. J. Geophys. Res. 104, 14739-14754.

Wolf-Gladrow, D. A., Neubauer, F. M., Lussem, M., Sep. 1987. Io’s interaction with the plasma torus - A self-consistent model. J. Geophys. Res. 92, 9949-9961.

Wolven, B. C., Moos, H. W., Retherford, K. D., Feldman, P. D., Strobel, D. F., Smyth, W. H., Roesler, F. L., Nov. 2001. Emission profiles of neutral oxygen and sulfur in Io's exospheric corona. J. Geophys. Res. 106, 2615526182.

Wong, M. C., Smyth, W. H., Jul. 2000. Model Calculations for Io's Atmosphere at Eastern and Western Elongations. Icarus 146, 60-74.

Zatsarinny, O., Tayal, S. S., Jun. 2002. Electron impact collision strengths and rates for neutral sulphur using the B-spline R-matrix approach. Journal of Physics B Atomic Molecular Physics 35, 2493-2503.

Zhang, J., Goldstein, D. B., Varghese, P. L., Gimelshein, N. E., Gimelshein, S. F., Levin, D. A., May 2003. Simulation of gas dynamics and radiation in volcanic plumes on Io. Icarus 163, 182-197. 


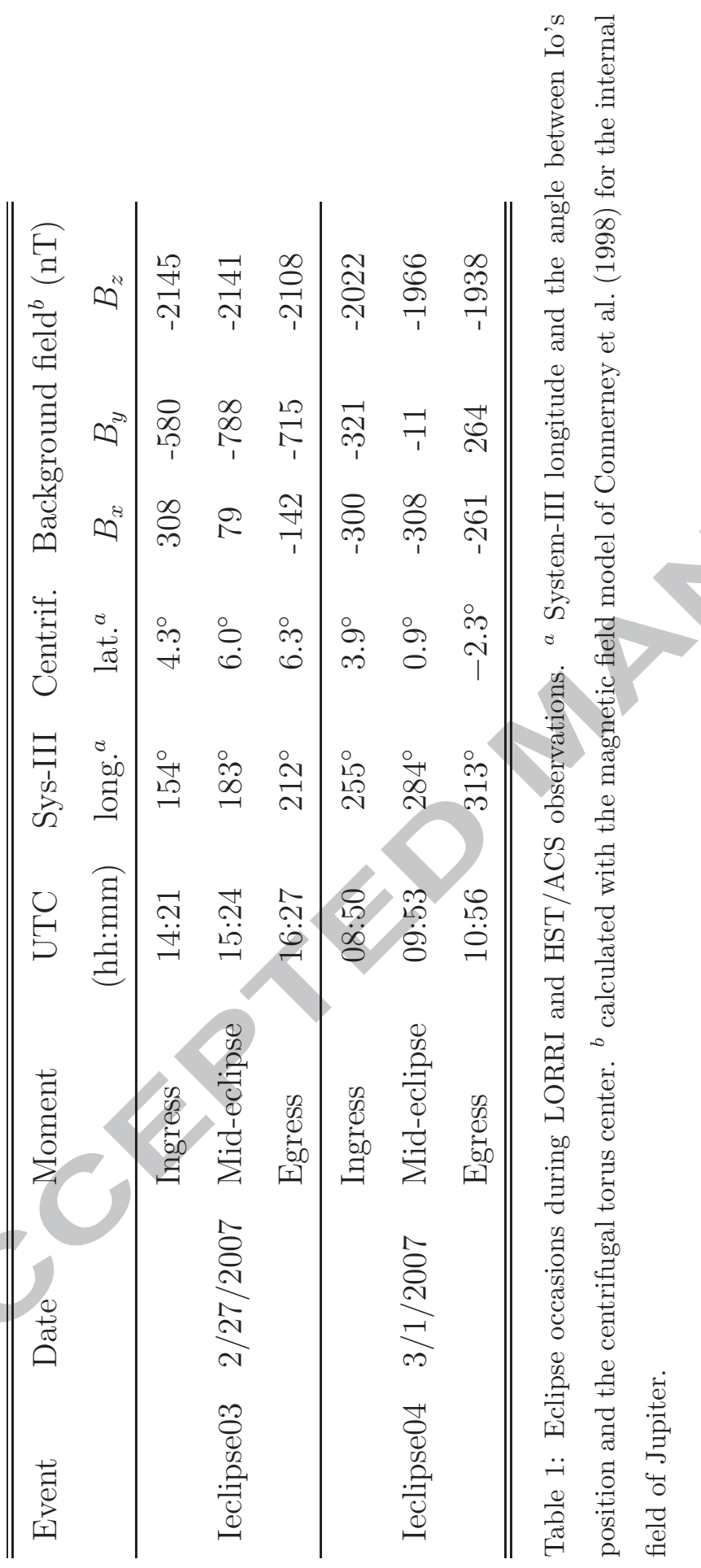




\begin{tabular}{lcccc}
\hline \hline Observation & $\begin{array}{c}\text { Bandpass } \\
(\mathrm{nm})\end{array}$ & $\begin{array}{c}\text { Resol. } \\
(\mathrm{km} / \text { pixel })\end{array}$ & $\begin{array}{c}\text { Viewing } \\
\text { geometry }^{a}\end{array}$ & Figure \\
\hline LORRI Ieclipse04 & $350-850$ & $\sim 56$ & $3^{\circ} \mathrm{S} 240^{\circ} \mathrm{W}$ & $3 \mathrm{a}$ \\
LORRI Ieclipse03 & $350-850$ & $\sim 15$ & $7^{\circ} \mathrm{S} \mathrm{310^{ \circ } \mathrm { W }}$ & $3 \mathrm{~b}$ \\
HST/ACS Ieclipse03 & $125-190$ & $\sim 135$ & $\sim 0^{\circ} 344^{\circ} \mathrm{W}$ & $3 \mathrm{c}$ \\
\hline \hline
\end{tabular}

Table 2: Summary of the $2007 \mathrm{NH} / \mathrm{LORRI}$ and HST/ACS eclipse observations. The observations are displayed in Figures 3a-c. All observations are combinations of several exposures during one eclipse event, as reported in Spencer et al. (2007b) . ${ }^{a}$ The viewing geometry of the respective observer (New Horizons, HST) refers to the mid-eclipse time and can thus differ for single exposures. 


\begin{tabular}{lccc}
\hline \hline Emitter & Sunlight observations & Eclipse observations & Eclipse simulation \\
& $I_{\text {sun }}(\mathrm{kR})$ & $I_{\text {ecl }}(\mathrm{kR})$ & $I_{\text {ecl,sim }}(\mathrm{kR})$ \\
\hline OI 135.6 & $0.48-0.88^{a}$ & $0.38-0.80^{a}$ & $0.4-0.7$ \\
OI 630.0/636.4 & - & $6.8^{f} / 14.2^{b} / 23.7^{c}$ & $6.2-11.8$ \\
SI 147.9 & $0.60-1.17^{a}$ & $0.43-1.20^{a}$ & $0.6-1.1$ \\
SI 190.0 & $1.68-2.33^{d}$ & - & $0.5-0.9$ \\
NaI 588.9/589.6 & - & $4^{b} / 6.9^{c}$ & $3.4-6.6$ \\
KI 767.0 & - & $<10.35^{e}$ & $2.2-4.3$ \\
\hline \hline
\end{tabular}

Table 3: Observed and simulated total intensities of the investigated emission lines. The variation of the simulated intensity results from changes in the viewing geometry as well as various upstream electron densities $\left(n_{e}=1900-3600 \mathrm{~cm}^{-3}\right)$. ${ }^{a}$ Retherford et al. (2007), ${ }^{b}$ Retherford (2002), ${ }^{c}$ Bouchez et al. (2000), ${ }^{d}$ Ballester et al. (1987), ${ }^{e}$ Geissler et al. (2004), ${ }^{f}$ Geissler et al. (1999) 


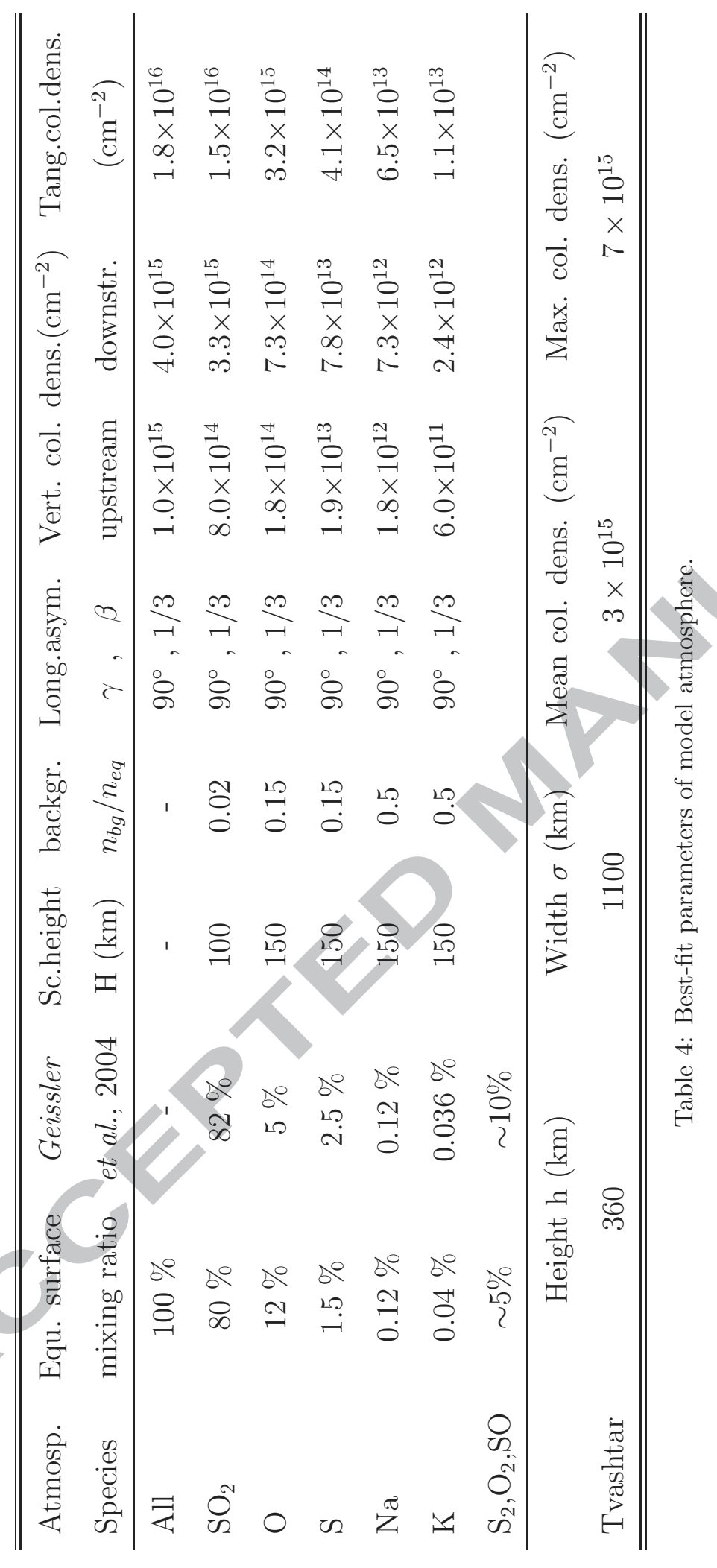




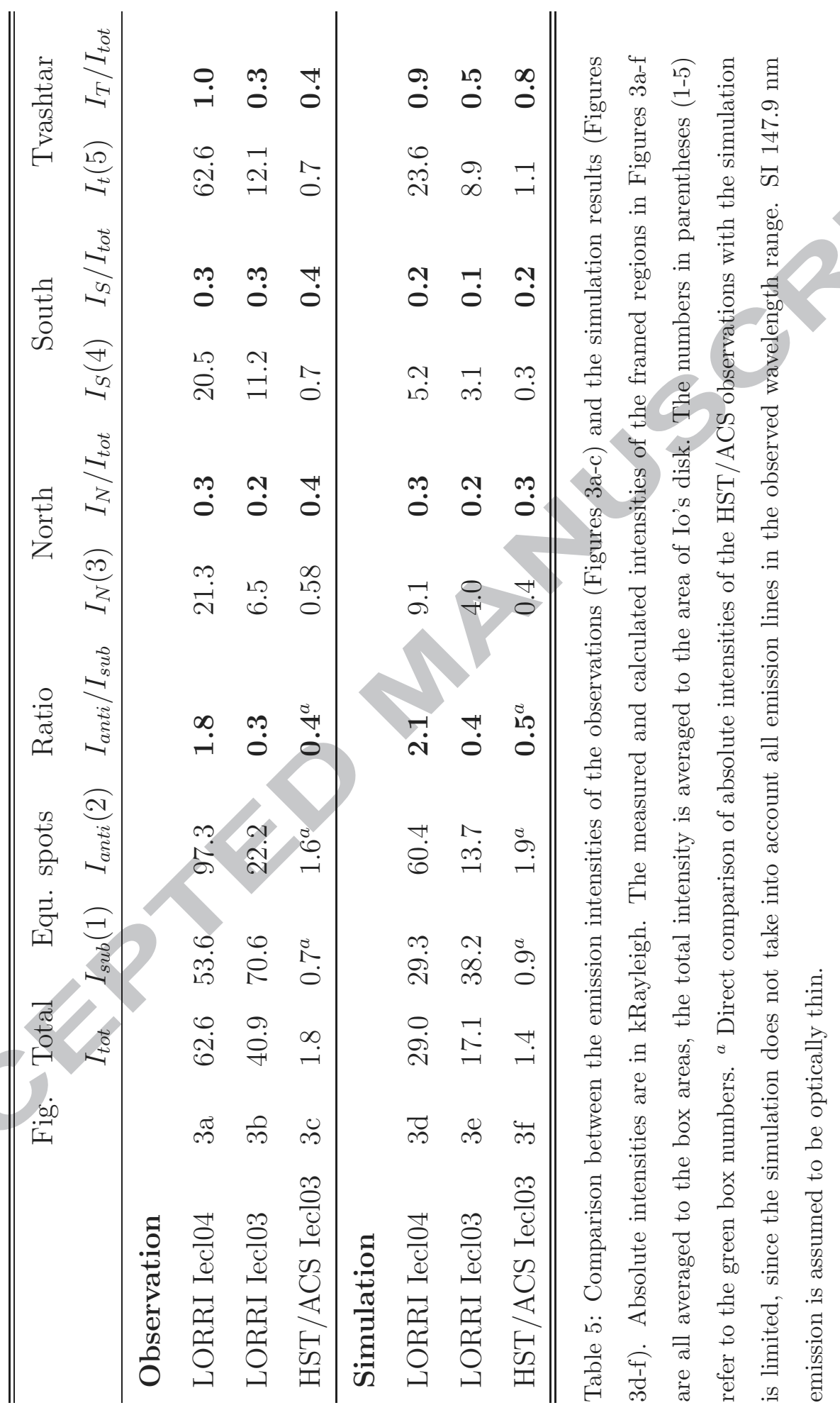


Figure 1: Observations of Io's auroral emission. (a) Multispectral eclipse image of Io taken by Cassini on January 1, 2001 (Geissler et al., 2004). Near-UV emission displayed in blue is attributed to $\mathrm{SO}_{2}$, emission in the red visible wavelength range (red in image) is primary from atomic oxygen (OI630.0 nm). (b) HST/STIS OI $135.6 \mathrm{~nm}$ image of October 14, 1997 (Roesler et al., 1999). 
Figure 2: Geometry of the Ieclipse03 and Ieclipse04 observations in the planetocentric coordinate system of Io. The black arrows point out the viewing longitude of the New Horizons probe and the Hubble Space Telescope, respectively. The jovian background field vectors in the $x y$ (orbital) plane at mid-eclipse are shown with purple arrows. Up to the right a $B$ field vector of $500 \mathrm{nT}$ is displayed for comparison $\left(B_{z} \approx 2000 \mathrm{nT}\right)$. In our simulation model the undisturbed plasma flow is directed in positive $x$ direction and the background field is assumed constant on negative $z$ direction (perpendicular to the displayed $x y$ plane). The expected key features of the aurora (very simplified) are marked with the red areas. 
Figure 3: Aurora observations and the respective simulated emission patterns. Properties of the New Horizons LORRI images (a and b) (Spencer et al., 2007a,b) and the HST/ACS image (c) (Retherford et al., 2007) are listed in Table 2. The displayed LORRI images have undergone some corrections to remove blemish emission. The corresponding simulated emission morphologies (d, e and f) are displayed with the respective viewing geometry. The color scale and the contour lines differ by a factor of 2 between observation and simulation for the LORRI images. Contours are $150 \mathrm{kR}$ (a), $100 \mathrm{kR}$ (b), $75 \mathrm{kR}$ (d) and $50 \mathrm{kR}$ (e). No contours in (c) and (f). Note that the HST/ACS observation (c) covers wavelengths from $125-190 \mathrm{~nm}$, whereas the corresponding simulation (f) takes into account only emission from OI $135.6 \mathrm{~nm}$ and SI $147.9 \mathrm{~nm}$. The orbital trailing $\left(270^{\circ}\right)$ longitudes are indicated with dashed meridians, the sub-jovian $\left(0^{\circ}\right)$ and anti-jovian $\left(180^{\circ}\right)$ meridians are displayed in plain bold. The emission within the green framed areas is investigated quantitatively. The total and relative intensities of the boxes are shown in Table 5 . 
Figure 4: Evolution of the electron temperature in the simulation model. The flux-tubes parallel to $\vec{B}_{0}(\| z)$ convect through the Io's interaction region given by the $2 \mathrm{D}$ plasma flow profile in the $x y$ plane. The heat conduction perpendicular to $\vec{B}$ is neglected, the heat conduction parallel to $\vec{B}$ is infinite outside the atmosphere. Inside the atmosphere the plasma cools down to $T_{i n}$ and the heat flow from outside $\left(T_{\text {out }}\right)$ is limited due to a finite heat conductivity along $\vec{B}$. The heat flow from the outer tube part to the inner is parametrized and depends on the temperature gradient between the two parts $\left(\sim\left(T_{\text {out }}-T_{i n}\right) / R_{\text {typ }}\right)$. 
Figure 5: The density distribution model for the Tvashtar plume displayed in a vertical plane through the center. The local neutral gas density is color-coded, the dashed line represents the derived vertical column density above the plume. The plume density is given by equation 8 and consists of three components: (1) the main plume, (2) a low density ring as found by ballistic models and (3) a high density region above the crater. The shape replicates roughly the modeled Pele-like plume by Zhang et al. (2003) with a peak column density over the plume center and a shallow decrease of the column density from $\sim 200$ to $\sim 500 \mathrm{~km}$ distance from the center. The plume-averaged column density is determined within a distance to the plume center of $<700 \mathrm{~km}$ (dashed-dotted vertical lines). 
Figure 6: Results of the plasma interaction simulation: (a) Electron temperature within the atmosphere in $x y$ plane. Isolines for 1 to $5 \mathrm{eV}$. (b) Electron density in units of torus density $\left(1900 \mathrm{~cm}^{-3}\right)$ in the equatorial plane at $z=0$. (c) Isolines of electric currents in $10^{-7} \mathrm{~A} \mathrm{~m}^{-2}$ in the northern Alfvén wing. 
Figure 7: Solid line: Modeled brightness ratio of the anti-jovian/sub-jovian spots. The brightnesses refer to the average emission intensity within a $\sim 800 \times 1100 \mathrm{~km}^{2}$ box located at the equatorial limb (see Figures 3). A spot brightness ratio derived from UV observations in sunlight by Retherford (2002) is shown $(+)$ for observing longitudes between $59^{\circ}$ and $74^{\circ}$ and between $241^{\circ}$ and $297^{\circ}$. Dotted: The longitudinal variation of the total intensity of OI 135.6 emission normalized to its minimum. 


\section{ACCEPTED MANUSCRIPT}

\section{a}

b
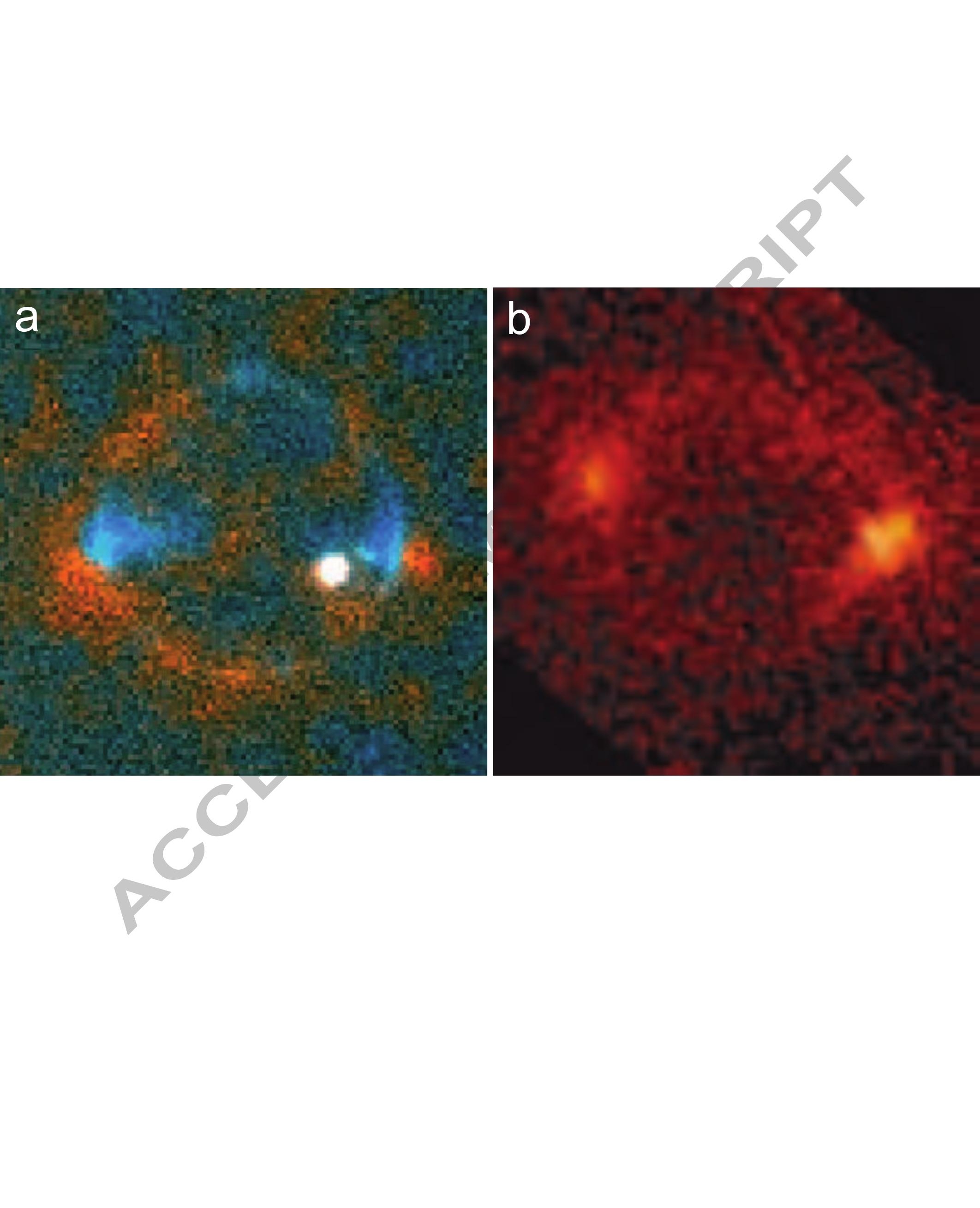

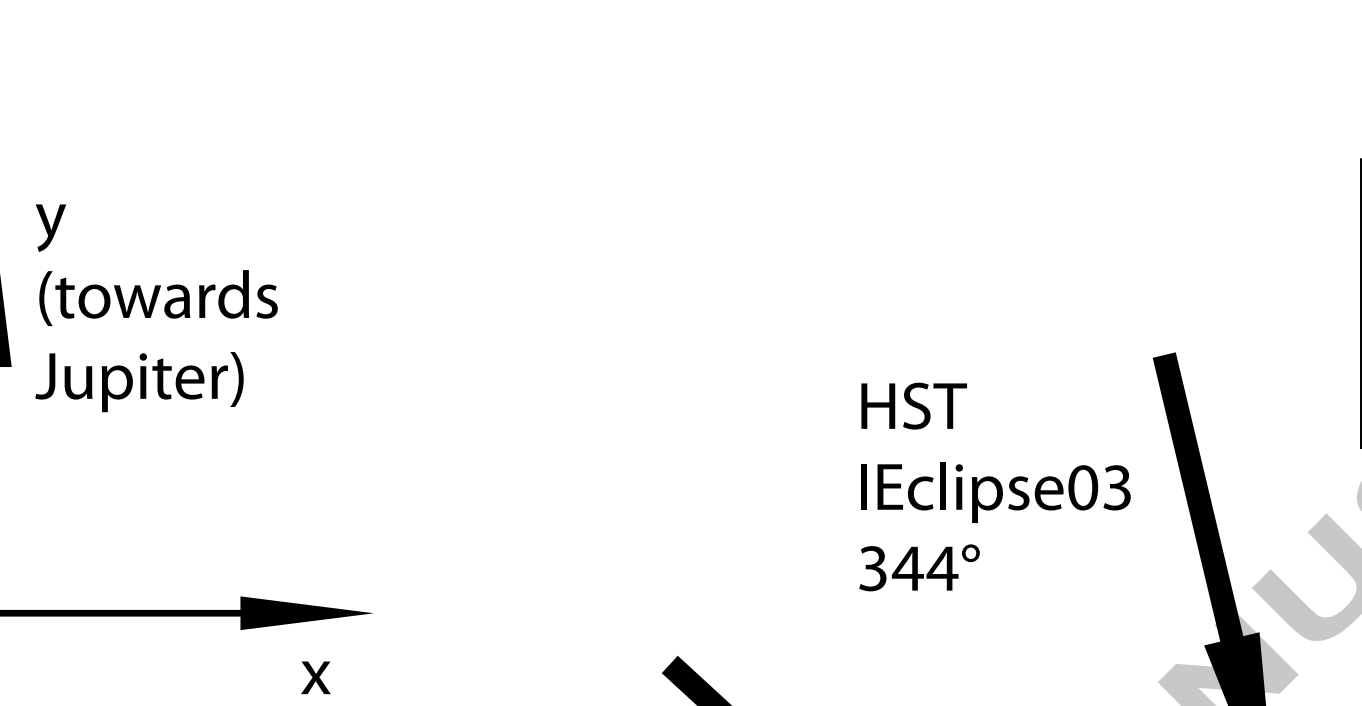

New Horizons IEclipse03 $\sim 310^{\circ}$

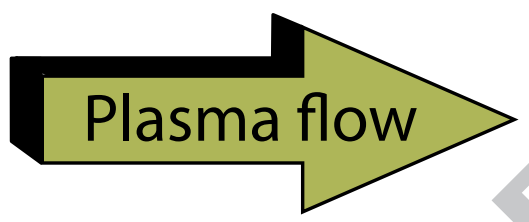

\section{$\otimes B_{\text {Model }}$}

Aurora B field vector feature $\quad(500 \mathrm{nT})$
Sub-Jovian spot
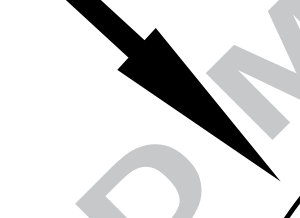

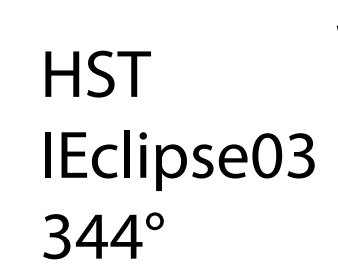
$344^{\circ}$

\section{New Horizons \\ IEclipse04 $\sim 240^{\circ}$ .}

$P$
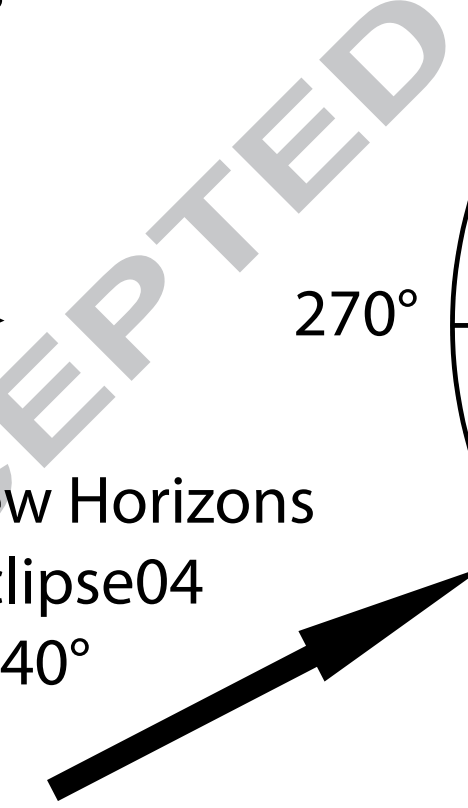

Anti-Jovian spot 

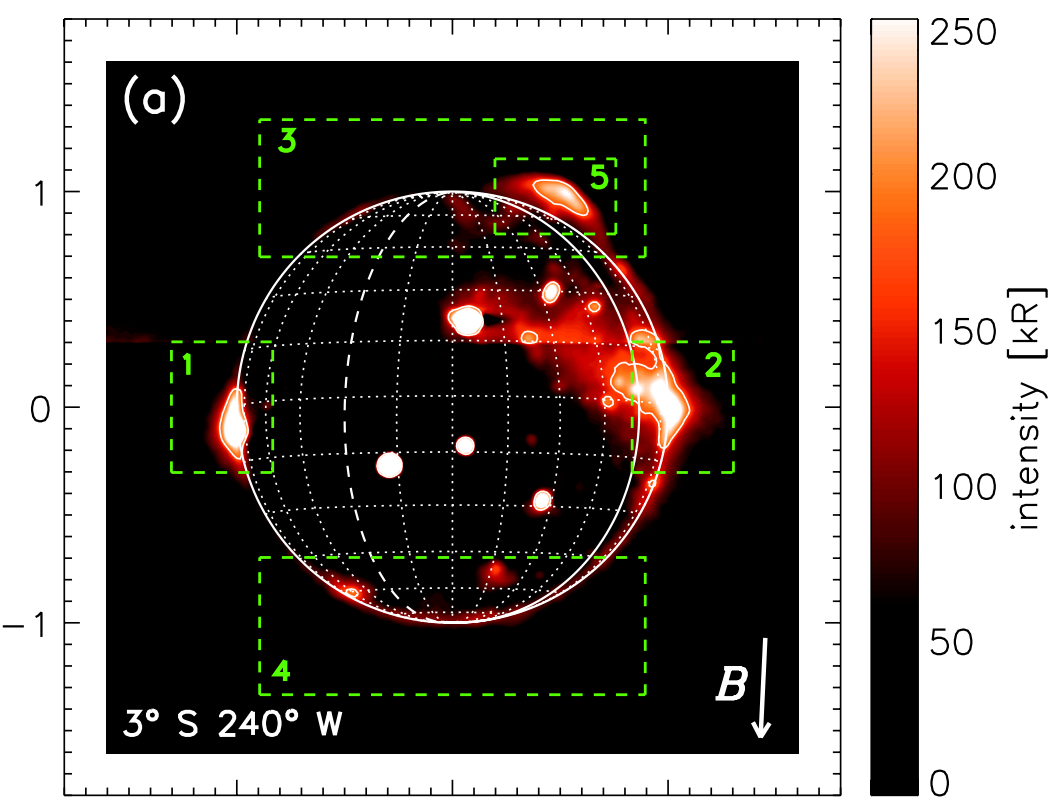

200

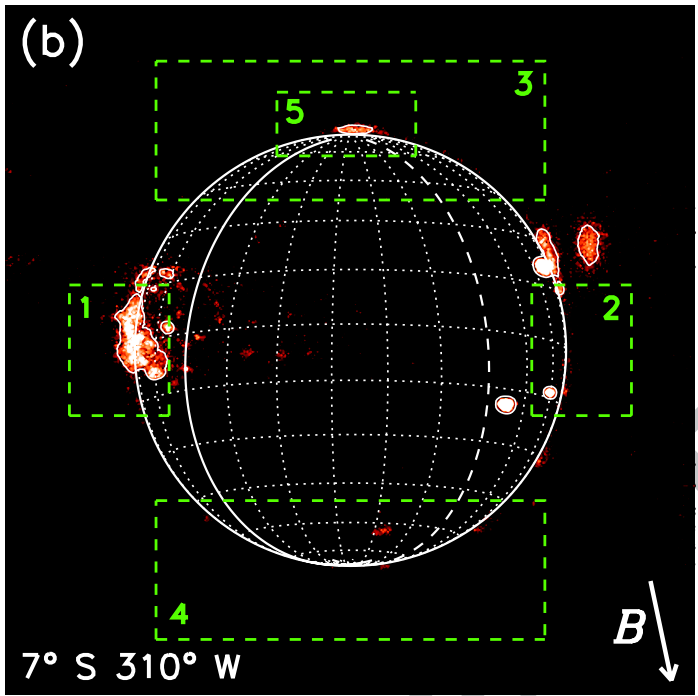

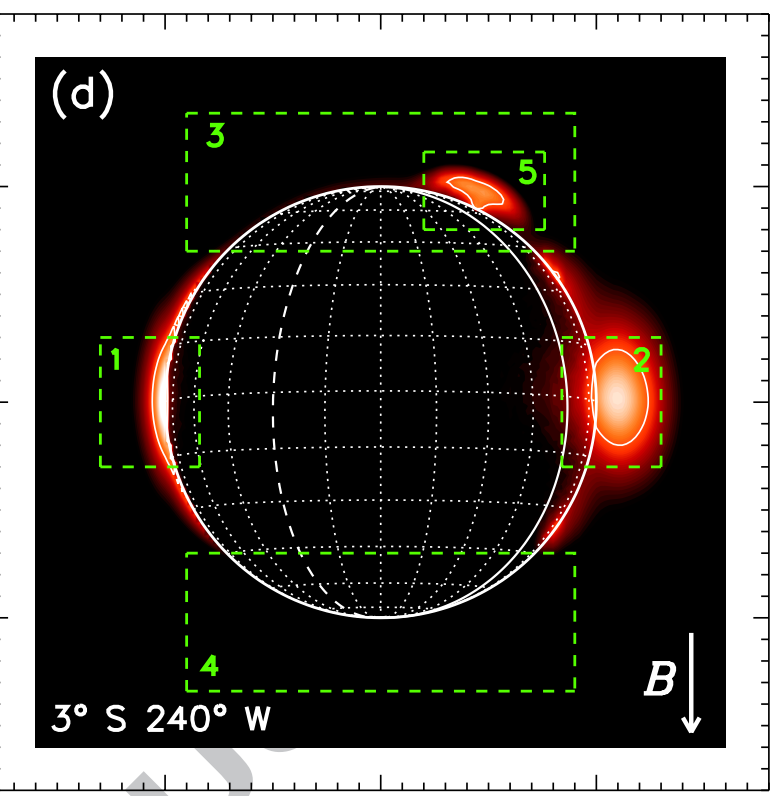
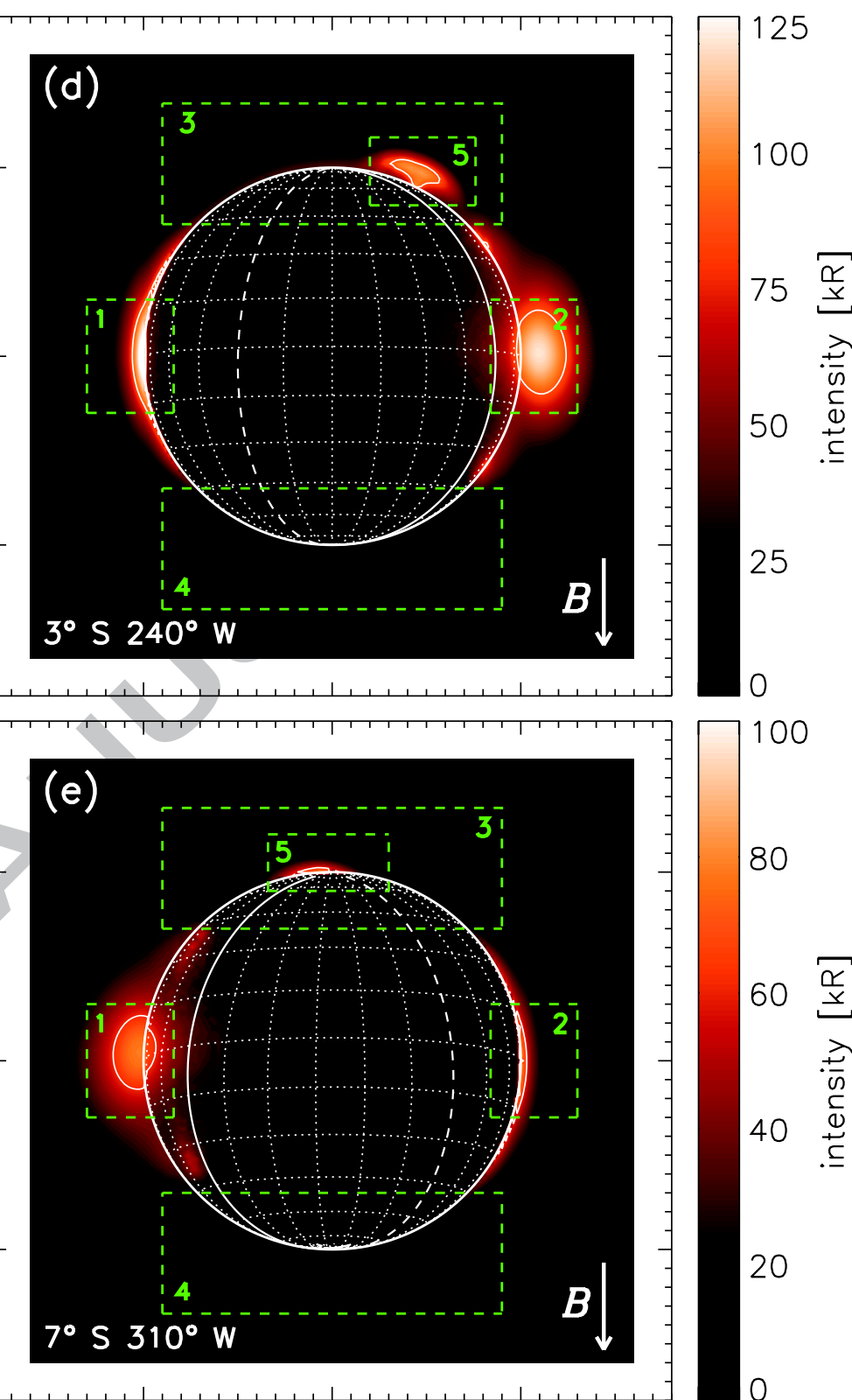

50

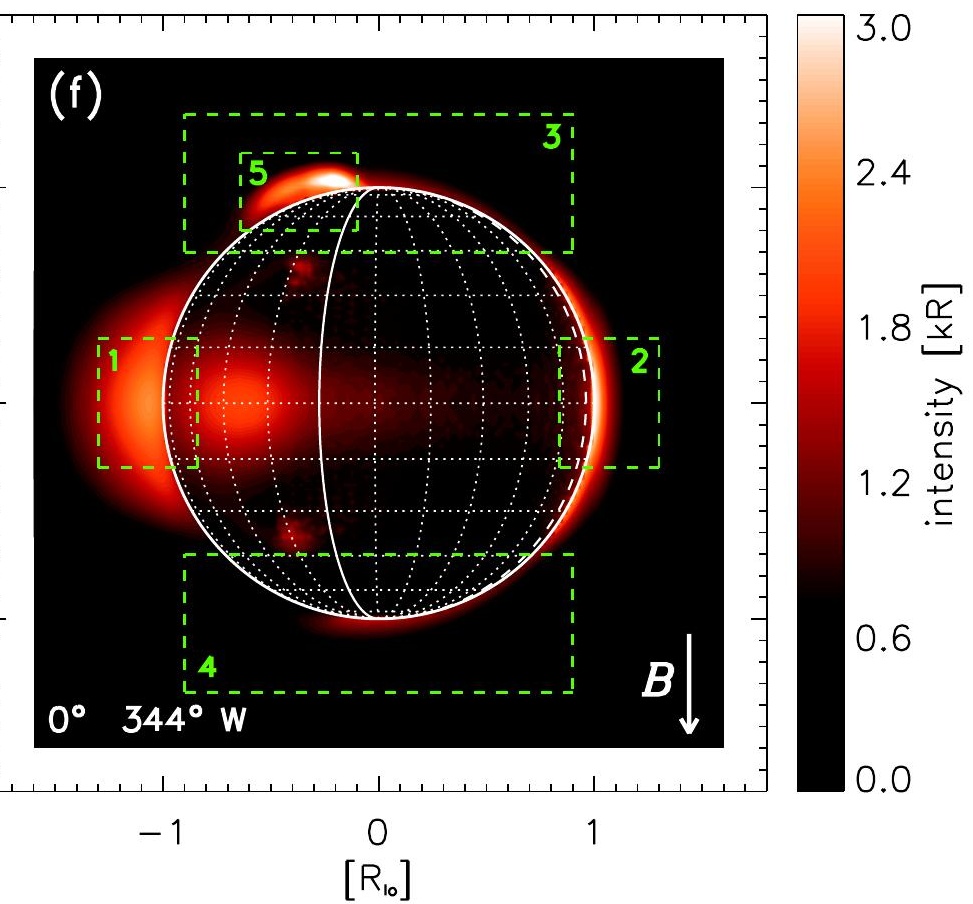



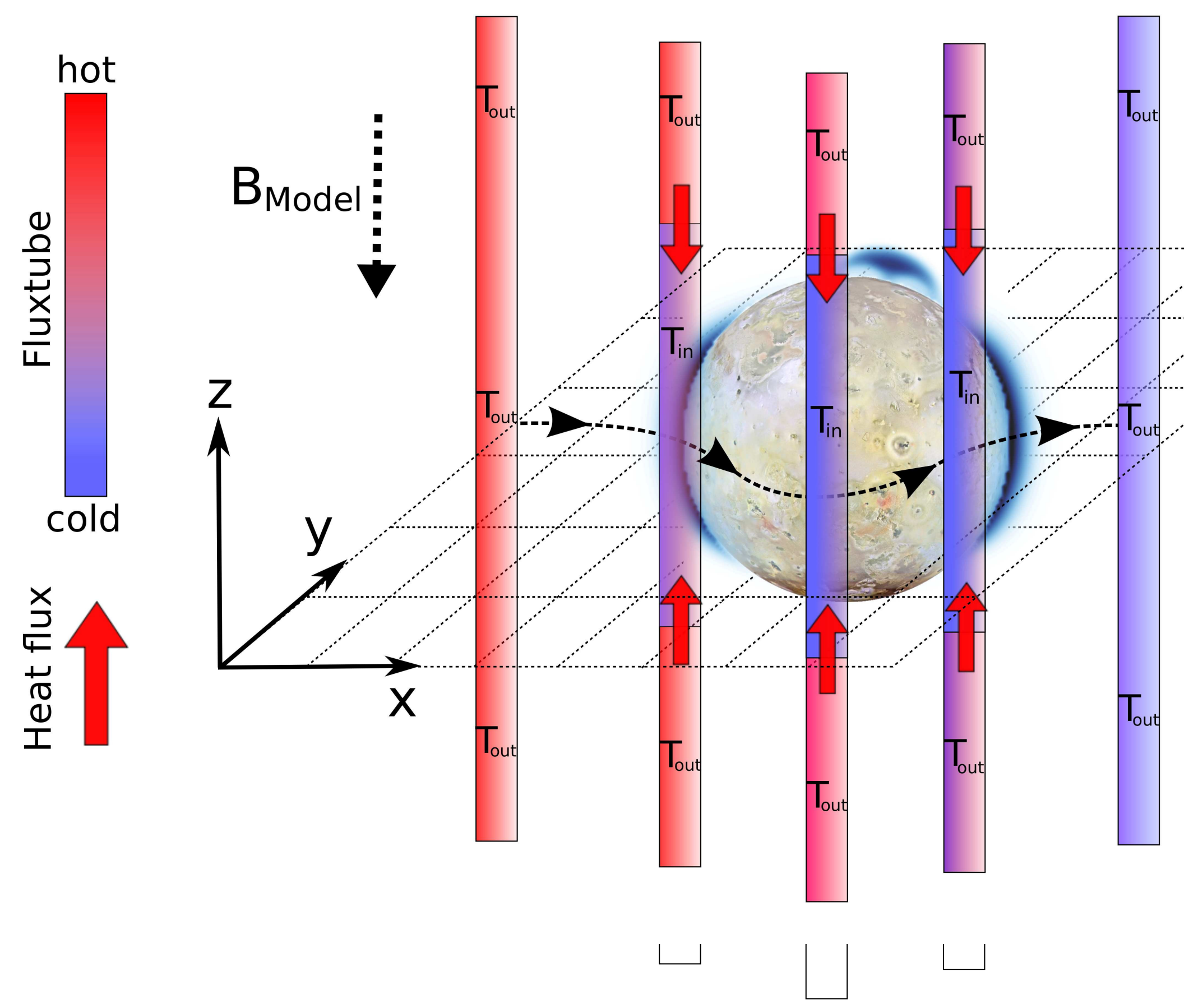


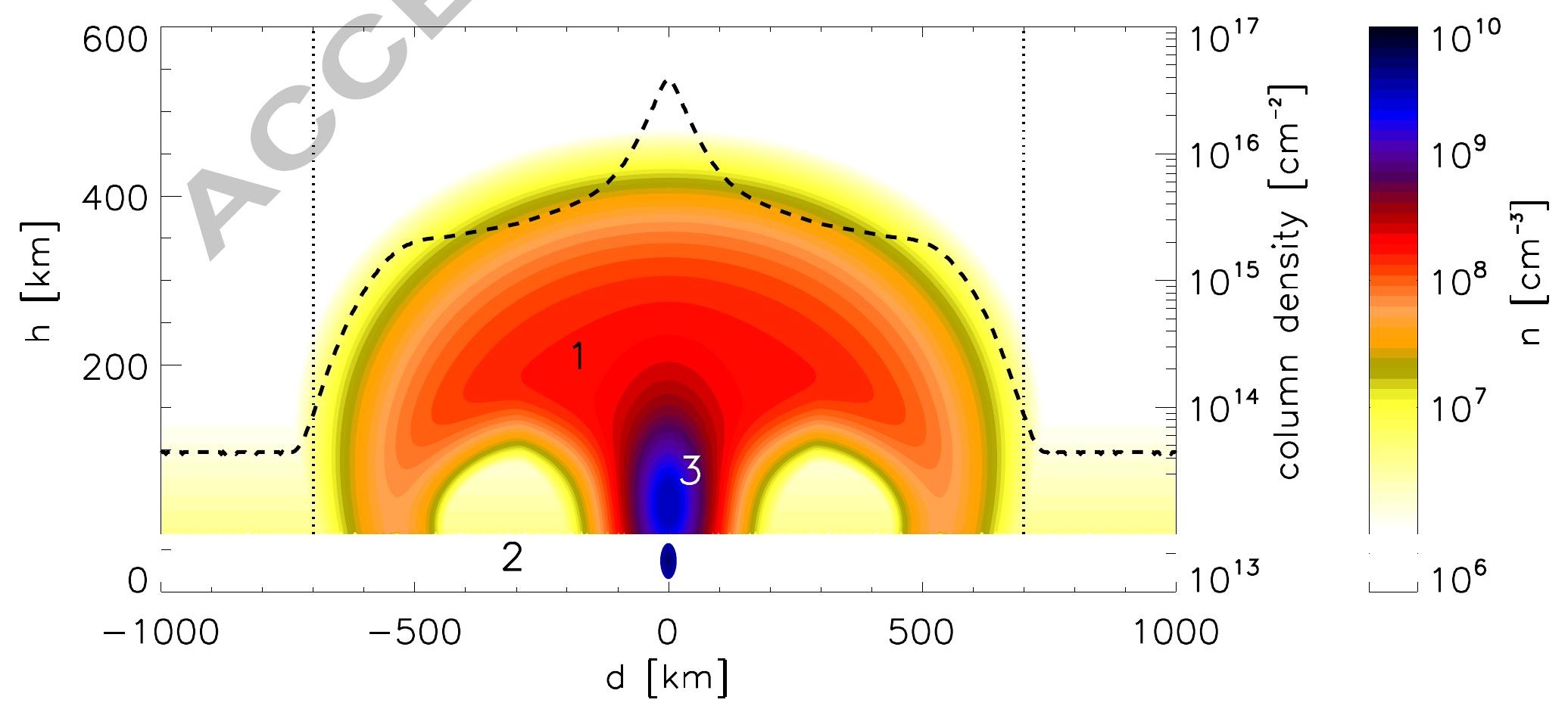




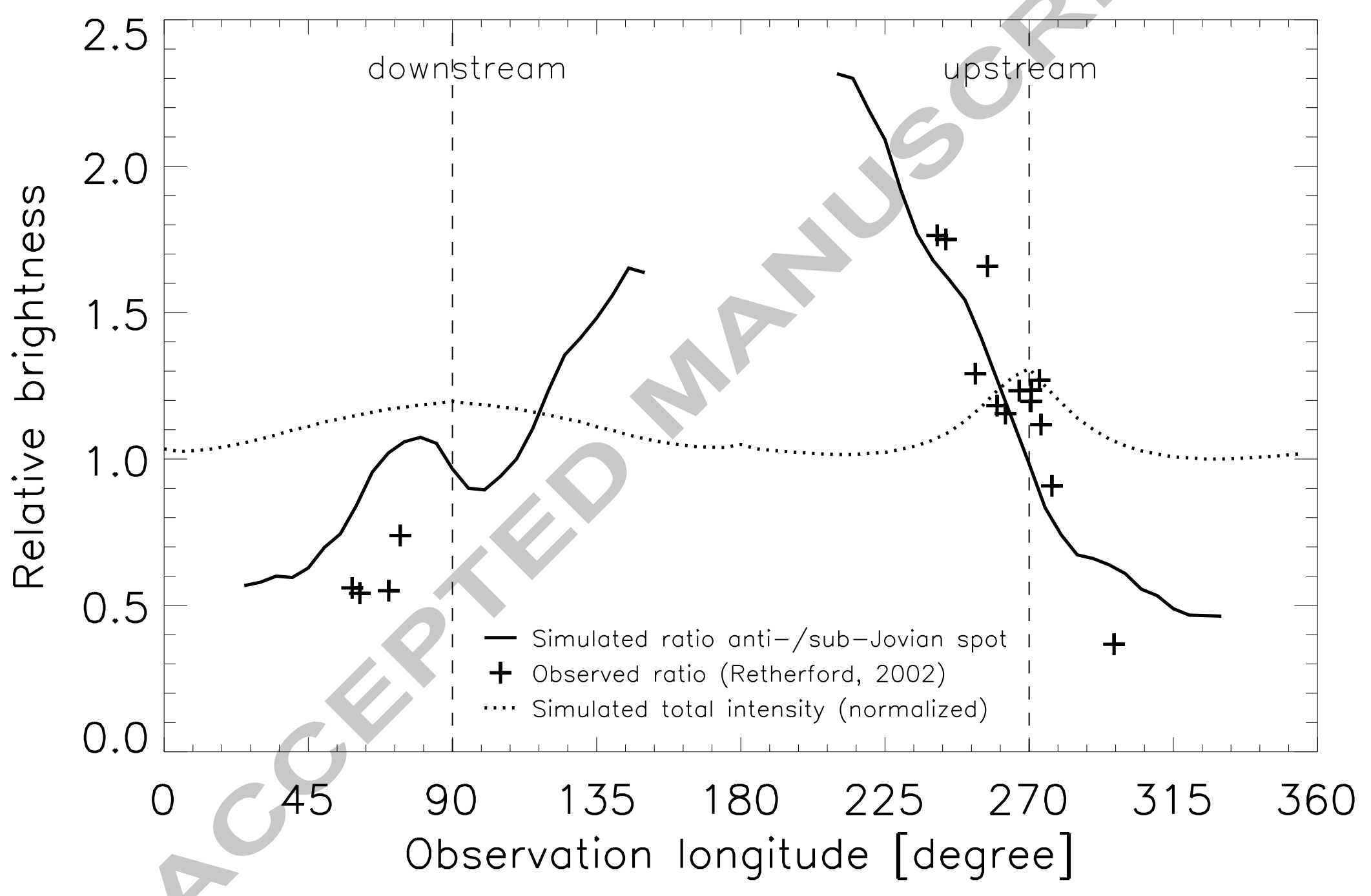

